\title{
OPTICAL SOLITONS IN BIREFRINGENT FIBERS BY EXTENDED TRIAL EQUATION METHOD
}

\author{
Mohammad Mirzazadeh ${ }^{1}$, Mehmet Ekici ${ }^{2}$ Abdullah Sonmezoglu ${ }^{2}$, Qin Zhou ${ }^{3}$, \\ Houria Triki ${ }^{4}$, Seithuti P. Moshokoa ${ }^{5}$, Anjan Biswas ${ }^{5,6} \&$ Milivoj Belic ${ }^{7}$ \\ ${ }^{1}$ Department of Engineering Sciences, Faculty of Technology and Engineering, \\ East of Guilan, University of Guilan, PC 44891-63157 Rudsar-Vajargah, Iran \\ 2 Department of Mathematics, Faculty of Science and Arts, \\ Bozok University, 66100 Yozgat, Turkey \\ ${ }^{3}$ School of Electronics and Information Engineering \\ Wuhan Donghu University, Wuhan-430212, P. R. China \\ ${ }^{4}$ Radiation Physics Laboratory, Department of Physics, Faculty of Sciences, \\ Badji Mokhtar University, PO Box 12, 23000 Annaba, Algeria \\ ${ }^{5}$ Department of Mathematics and Statistics \\ Tshwane University of Technology, Pretoria-0008, South Africa \\ 6 Department of Mathematics, \\ King Abdulaziz University, Jeddah-21589, Saudi Arabia \\ 7 Science Program, Texas A \& M University at Qatar \\ PO Box 23874, Doha, Qatar
}




\begin{abstract}
This paper studies solitons in optical fibers by the aid of extended trial function method. This powerful mathematical tool is applied to birefringent fibers with Kerr and parabolic laws of nonlinearity. Bright and singular soliton solutions are obtained along with respective constraints that must hold to guarantee the existence of these solitons. As a byproduct, singular periodic and doubly periodic solutions are also recovered from this algorithm.
\end{abstract}

Key words: birefringence; solitons; extended trial equation method.

\title{
1 INTRODUCTION
}

Optical solitons is one of the most fascinating areas of research in the field of nonlinear optics. In fact, solitons are treasure-trove for long distance fiber-optic communication along trans-continental and trans-oceanic distances. However, when rubber meets the road, there are several problems one encounters. These are pulse dissipation and attenuation, evolution of ghost pulses, collision-induced frequency and timing jitter and many more. This paper will discuss the consequence of pulse splitting. When a pulse is injected into the optical fiber at the initial end, it tends to split into two orthogonal components. This occurs due to random variation of fiber diameter, twists and bends as well as other unwanted features that naturally cause these polarization. The splitting of pulses lead to differential group delay and this phenomena is called birefringence in optical fibers.

The basic fabric of soliton propagation is the nonlinear Schrödinger's equation (NLSE) [1-20]. For birefringent fibers, the dynamics of these soliton propagation is modeled by vector coupled NLSE where the two components of NLSE represent split pulse components. The coupling between the two components is maintained by cross-phase modulation. There are several perturbation terms that are considered in this paper, although four-wave mixing (FWM) terms are neglected. Two kinds of optical fibers are studied in this paper. These are Kerr law nonlinearity and parabolic law nonlinear medium.

The aim of this paper is to extract exact 1-soliton solution for the coupled NLSE by using a powerful integration tool. It is the extended trial equation method. This algorithm extracts bright and singular soliton solutions in birefringent fibers along with respective constraint conditions that guarantee the existence of these solitons. This integration scheme, as a byproduct, also reveals additional solutions such as singular-periodic and doubly periodic solutions that are not studied in nonlinear optics. The details are discussed in the upcoming sections.

\section{KERR LAW NONLINEARITY}

This section bright and singular soliton solutions in birefringent optical fibers with Kerr law nonlinearity. The dimensionless form of the coupled NLSE with Kerr law nonlinearity is given by $[1,2]$

$$
\begin{aligned}
& i q_{t}+a_{1} q_{x x}+b_{1} q_{x t}+\left(c_{1}|q|^{2}+d_{1}|r|^{2}\right) q+i\left\{\alpha_{1} q_{x}+\beta_{1}\left(|q|^{2} q\right)_{x}+\nu_{1}\left(|q|^{2}\right)_{x} q+\theta_{1}|q|^{2} q_{x}+\gamma_{1} q_{x x x}\right\}=0, \\
& i r_{t}+a_{2} r_{x x}+b_{2} r_{x t}+\left(c_{2}|r|^{2}+d_{2}|q|^{2}\right) r+i\left\{\alpha_{2} r_{x}+\beta_{2}\left(|r|^{2} r\right)_{x}+\nu_{2}\left(|r|^{2}\right)_{x} r+\theta_{2}|r|^{2} r_{x}+\gamma_{2} r_{x x x}\right\}=0 .
\end{aligned}
$$

In (1) and (2), $q(x, t)$ and $r(x, t)$ are complex valued functions that represents the soliton profiles for the two components in birefringent fibers. For $l=1,2, a_{l}$ represents group-velocity dispersion (GVD) and $b_{l}$ is the coefficient of spatio-temporal dispersion (STD) along the two components. These STD terms are necessary to be included, since it induces the model to be well-posed as reported during $2012[8,9]$. However, NLSE with STD was already studied in the past with STD [18]. Next, $c_{l}$ and $d_{l}$ represents the self-phase modulation (SPM) and cross-phase modulation 
(XPM) respectively. From perturbation terms $\alpha_{l}$ represents the inter-modal dispersion, $\beta_{l}$ is the self-steepening term, $\nu_{l}$ and $\theta_{l}$ are nonlinear dispersions and finally $\gamma_{l}$ is the third order dispersion that must be included for small GVD.

The extended trial function method method $[10-15,17]$ is implemented. To start off, $q(x, t)$ and $r(x, t)$ are written in the phase-amplitude format as $[1,2]$

$$
q(x, t)=P_{1}(x, t) e^{i \phi_{1}(x, t)}=P_{1}(x, t) e^{i\left(-\kappa_{1} x+\omega_{1} t+\sigma_{1}\right)},
$$

and

$$
r(x, t)=P_{2}(x, t) e^{i \phi_{2}(x, t)}=P_{2}(x, t) e^{i\left(-\kappa_{2} x+\omega_{2} t+\sigma_{2}\right)},
$$

where $P_{l}$ for $l=1,2$ are the amplitude components of the solitons and $\phi_{l}(x, t)$ are its phase components, defined as

$$
\phi_{l}(x, t)=-\kappa_{l} x+\omega_{l} t+\sigma_{l} .
$$

Here, $\kappa_{l}$ are the frequencies of the solitons in each of the two components, $\omega_{l}$ are the wave numbers, while $\sigma_{l}$ are the phase constants. Substituting (3) and (4) into (1) and (2) and decomposing into real and imaginary parts lead to

$$
\left(\omega_{l}+a_{l} \kappa_{l}^{2}-b_{l} \kappa_{l} \omega_{l}-\alpha_{l} \kappa_{l}+\gamma_{l} \kappa_{l}^{3}\right) P_{l}-d_{l} P_{l} P_{\bar{l}}^{2}-\left(\beta_{l} \kappa_{l}+\theta_{l} \kappa_{l}+c_{l}\right) P_{l}^{3}-\left(a_{l}+3 \gamma_{l} \kappa_{l}\right) \frac{\partial^{2} P_{l}}{\partial x^{2}}-b_{l} \frac{\partial^{2} P_{l}}{\partial x \partial t}=0,
$$

and

$$
\left(1-b_{l} \kappa_{l}\right) \frac{\partial P_{l}}{\partial t}-\left(2 a_{l} \kappa_{l}-b_{l} \omega_{l}-\alpha_{l}+3 \gamma_{l} \kappa_{l}^{2}\right) \frac{\partial P_{l}}{\partial x}+\left(3 \beta_{l}+2 \nu_{l}+\theta_{l}\right) P_{l}^{2} \frac{\partial P_{l}}{\partial x}+\gamma_{l} \frac{\partial^{3} P_{l}}{\partial x^{3}}=0,
$$

for $l=1,2$ and $\bar{l}=3-l$. Under the traveling wave transformation

$$
P_{1}(x, t)=U_{1}(\xi), \quad P_{2}(x, t)=U_{2}(\xi), \quad \xi=B(x-v t),
$$

we have

$$
\left(\omega_{l}+a_{l} \kappa_{l}^{2}-b_{l} \kappa_{l} \omega_{l}-\alpha_{l} \kappa_{l}+\gamma_{l} \kappa_{l}^{3}\right) U_{l}-d_{l} U_{l} U_{\bar{l}}^{2}-\left(\beta_{l} \kappa_{l}+\theta_{l} \kappa_{l}+c_{l}\right) U_{l}^{3}-\left(a_{l}-b_{l} v+3 \gamma_{l} \kappa_{l}\right) B^{2} \frac{d^{2} U_{l}}{d \xi^{2}}=0,
$$

and

$$
-\left(v\left(1-b_{l} \kappa_{l}\right)+2 a_{l} \kappa_{l}-b_{l} \omega_{l}-\alpha_{l}+3 \gamma_{l} \kappa_{l}^{2}\right) B \frac{d U_{l}}{d \xi}+\left(3 \beta_{l}+2 \nu_{l}+\theta_{l}\right) B U_{l}^{2} \frac{d U_{l}}{d \xi}+\gamma_{l} B^{3} \frac{d^{3} U_{l}}{d \xi^{3}}=0 .
$$

From (10), the third term implies

$$
\gamma_{l}=0
$$

for $l=1,2$ so that Eqs. (9) and (10) reduces to

$$
\left(\omega_{l}+a_{l} \kappa_{l}^{2}-b_{l} \kappa_{l} \omega_{l}-\alpha_{l} \kappa_{l}\right) U_{l}-d_{l} U_{l} U_{\bar{l}}^{2}-\left(\beta_{l} \kappa_{l}+\theta_{l} \kappa_{l}+c_{l}\right) U_{l}^{3}-\left(a_{l}-b_{l} v\right) B^{2} \frac{d^{2} U_{l}}{d \xi^{2}}=0
$$

and

$$
-\left(v\left(1-b_{l} \kappa_{l}\right)+2 a_{l} \kappa_{l}-b_{l} \omega_{l}-\alpha_{l}\right) B \frac{d U_{l}}{d \xi}+\left(3 \beta_{l}+2 \nu_{l}+\theta_{l}\right) B U_{l}^{2} \frac{d U_{l}}{d \xi}=0 .
$$

Therefore, exact solutions for Kerr law nonlinearity in birefringent fibers will exist provided the third order dispersion coefficient is zero. This is an important issue that was earlier reported in 2014 [1]. Next, setting the coefficients of the linearly independent functions, in (10), to zero gives

$$
v=\frac{2 a_{l} \kappa_{l}-b_{l} \omega_{l}-\alpha_{l}}{b_{l} \kappa_{l}-1}
$$


and

$$
3 \beta_{l}+2 \nu_{l}+\theta_{l}=0 .
$$

Eq. (14) is the velocity of the soliton for two components and Eq. (15) represents the constraint condition in order for the soliton to exist. Also, from (14), the following condition is needed:

$$
b_{l} \kappa_{l} \neq 1
$$

Then, from (14), equating the velocity of the solitons leads to a constraint condition given by

$$
\left(2 a_{1} \kappa_{1}-b_{1} \omega_{1}-\alpha_{1}\right)\left(b_{2} \kappa_{2}-1\right)=\left(2 a_{2} \kappa_{2}-b_{2} \omega_{2}-\alpha_{2}\right)\left(b_{1} \kappa_{1}-1\right) .
$$

Consequently, the real part Eq. (9) changes to

$$
\begin{aligned}
& \left(\omega_{1}+a_{1} \kappa_{1}^{2}-b_{1} \kappa_{1} \omega_{1}-\alpha_{1} \kappa_{1}\right) U_{1}-d_{1} U_{1} U_{2}^{2}-\left(\beta_{1} \kappa_{1}+\theta_{1} \kappa_{1}+c_{1}\right) U_{1}^{3}-\left(a_{1}-b_{1} v\right) B^{2} \frac{d^{2} U_{1}}{d \xi^{2}}=0, \\
& \left(\omega_{2}+a_{2} \kappa_{2}^{2}-b_{2} \kappa_{2} \omega_{2}-\alpha_{2} \kappa_{2}\right) U_{2}-d_{2} U_{2} U_{1}^{2}-\left(\beta_{2} \kappa_{2}+\theta_{2} \kappa_{2}+c_{2}\right) U_{2}^{3}-\left(a_{2}-b_{2} v\right) B^{2} \frac{d^{2} U_{2}}{d \xi^{2}}=0 .
\end{aligned}
$$

We will now analyze the system of equations (18) and (19) to secure soliton solutions by extended trial equation method in the following subsection.

\subsection{EXTENDED TRIAL EQUATION METHOD}

For solutions to (18) and (19), the following assumption for the soliton structure is made

$$
\begin{aligned}
& U_{1}=\sum_{i=0}^{\varsigma} \tau_{i} \Psi^{i}, \\
& U_{2}=\sum_{i=0}^{\tilde{\varsigma}} \tilde{\tau}_{i} \Psi^{i},
\end{aligned}
$$

where

$$
\left(\Psi^{\prime}\right)^{2}=\Lambda(\Psi)=\frac{\Phi(\Psi)}{\Upsilon(\Psi)}=\frac{\mu_{\epsilon} \Psi^{\epsilon}+\cdots+\mu_{1} \Psi+\mu_{0}}{\chi_{\rho} \Psi^{\rho}+\cdots+\chi_{1} \Psi+\chi_{0}} .
$$

Using the relations (20)-(22), we can derive the terms $U_{1}^{\prime \prime}$ and $U_{2}^{\prime \prime}$ as follows:

$$
U_{1}^{\prime \prime}=\frac{\Phi^{\prime}(\Psi) \Upsilon(\Psi)-\Phi(\Psi) \Upsilon^{\prime}(\Psi)}{2 \Upsilon^{2}(\Psi)}\left(\sum_{i=0}^{\varsigma} i \tau_{i} \Psi^{i-1}\right)+\frac{\Phi(\Psi)}{\Upsilon(\Psi)}\left(\sum_{i=0}^{\varsigma} i(i-1) \tau_{i} \Psi^{i-2}\right)
$$

and

$$
U_{2}^{\prime \prime}=\frac{\Phi^{\prime}(\Psi) \Upsilon(\Psi)-\Phi(\Psi) \Upsilon^{\prime}(\Psi)}{2 \Upsilon^{2}(\Psi)}\left(\sum_{i=0}^{\tilde{\varsigma}} i \tilde{\tau}_{i} \Psi^{i-1}\right)+\frac{\Phi(\Psi)}{\Upsilon(\Psi)}\left(\sum_{i=0}^{\tilde{\varsigma}} i(i-1) \tilde{\tau}_{i} \Psi^{i-2}\right),
$$

where $\Phi(\Psi)$ and $\Upsilon(\Psi)$ are polynomials of $\Psi$. We can reduce Eq. (22) to the elementary integral form as follows:

$$
\pm\left(\xi-\xi_{0}\right)=\int \frac{d \Psi}{\sqrt{\Lambda(\Psi)}}=\int \sqrt{\frac{\Upsilon(\Psi)}{\Phi(\Psi)}} d \Psi .
$$

We substitute Eqs. (20), (21), (23) and (24) into Eqs. (18) and (19). Then, we use the balance principle and find that

$$
\varsigma=\tilde{\varsigma}=\frac{\epsilon-\rho-2}{2} .
$$


When $\epsilon=4, \rho=0$ and $\varsigma=\tilde{\varsigma}=1$ in Eq. (26), we have

$$
\begin{aligned}
& U_{1}=\tau_{0}+\tau_{1} \Psi, \\
& U_{2}=\tilde{\tau}_{0}+\tilde{\tau}_{1} \Psi,
\end{aligned}
$$

and

$$
\begin{aligned}
& U_{1}^{\prime \prime}=\frac{\tau_{1}\left(4 \mu_{4} \Psi^{3}+3 \mu_{3} \Psi^{2}+2 \mu_{2} \Psi+\mu_{1}\right)}{2 \chi_{0}}, \\
& U_{2}^{\prime \prime}=\frac{\tilde{\tau}_{1}\left(4 \mu_{4} \Psi^{3}+3 \mu_{3} \Psi^{2}+2 \mu_{2} \Psi+\mu_{1}\right)}{2 \chi_{0}},
\end{aligned}
$$

where $\mu_{4} \neq 0, \chi_{0} \neq 0$. We substitute Eqs. (27)-(30) into Eqs. (18)-(19) and collect the coefficients of $\Psi$. Then, we solve the resulting system and obtain the following results:

$$
\begin{gathered}
\mu_{1}=\mp \frac{2 i \tau_{0} \mathcal{H}_{2}\left(\mu_{2} B^{2} \mathcal{H}_{1}^{2}+2 \tau_{0}^{2} \chi_{0} \mathcal{H}_{3}\right)}{\tilde{\tau}_{1} B^{2} \mathcal{H}_{1}^{3}}, \quad \mu_{3}=\mp \frac{2 i \tau_{0} \tilde{\tau}_{1} \chi_{0} \mathcal{H}_{3}}{B^{2} \mathcal{H}_{1} \mathcal{H}_{2}}, \quad \mu_{4}=-\frac{\tilde{\tau}_{1}^{2} \chi_{0} \mathcal{H}_{3}}{2 B^{2} \mathcal{H}_{4}}, \\
\mu_{0}=\mu_{0}, \quad \mu_{2}=\mu_{2}, \quad \chi_{0}=\chi_{0}, \quad \tau_{0}=\tau_{0}, \quad \tilde{\tau}_{1}=\tilde{\tau}_{1}, \quad \tau_{1}= \pm \frac{i \tilde{\tau}_{1} \mathcal{H}_{1}}{\mathcal{H}_{2}}, \quad \tilde{\tau}_{0}=\mp \frac{i \tau_{0} \mathcal{H}_{2}}{\mathcal{H}_{1}}, \\
\omega_{1}=\frac{\mu_{2} B^{2} \mathcal{H}_{1}^{2}\left(a_{1}-b_{1} v\right)+\chi_{0}\left[\kappa_{1} \mathcal{H}_{5}\left(a_{1} \kappa_{1}-\alpha_{1}\right)+3 \tau_{0}^{2} \mathcal{H}_{3}\left(a_{1}-b_{1} v\right)\right]}{\chi_{0} \mathcal{H}_{5}\left(b_{1} \kappa_{1}-1\right)}, \\
\omega_{2}=\frac{\mu_{2} B^{2}\left(a_{2}-b_{2} v\right)\left(\mathcal{H}_{6}-\mathcal{H}_{7}\right)+\chi_{0}\left[\kappa_{2}\left(\alpha_{2}-a_{2} \kappa_{2}\right)\left(\mathcal{H}_{6}-\mathcal{H}_{7}\right)-3 \tau_{0}^{2} \mathcal{H}_{3}\left(a_{2}-b_{2} v\right)\right]}{\chi_{0} \mathcal{H}_{1}^{2}\left(b_{2} \kappa_{2}-1\right)},
\end{gathered}
$$

where

$$
\begin{gathered}
\mathcal{H}_{1}=\sqrt{a_{1} c_{2}-a_{2} d_{1}-b_{1} c_{2} v+b_{2} d_{1} v+\kappa_{2}\left(a_{1}-b_{1} v\right)\left(\beta_{2}+\theta_{2}\right)}, \\
\mathcal{H}_{2}=\sqrt{d_{2}\left(a_{1}-b_{1} v\right)-a_{2}\left(c_{1}+\kappa_{1}\left(\beta_{1}+\theta_{1}\right)\right)+b_{2} v\left(c_{1}+\kappa_{1}\left(\beta_{1}+\theta_{1}\right)\right)}, \\
\mathcal{H}_{3}=-d_{1} d_{2}+\left(c_{1}+\kappa_{1}\left(\beta_{1}+\theta_{1}\right)\right)\left(c_{2}+\kappa_{2}\left(\beta_{2}+\theta_{2}\right)\right), \\
\mathcal{H}_{4}=a_{2} c_{1}-a_{1} d_{2}-b_{2} c_{1} v+b_{1} d_{2} v+\kappa_{1}\left(a_{2}-b_{2} v\right)\left(\beta_{1}+\theta_{1}\right), \\
\mathcal{H}_{5}=d_{1}\left(a_{2}-b_{2} v\right)-a_{1}\left(c_{2}+\kappa_{2}\left(\beta_{2}+\theta_{2}\right)\right)+b_{1} v\left(c_{2}+\kappa_{2}\left(\beta_{2}+\theta_{2}\right)\right), \\
\mathcal{H}_{6}=d_{1}\left(a_{2}-b_{2} v\right)+b_{1} v\left(c_{2}+\kappa_{2}\left(\beta_{2}+\theta_{2}\right)\right), \quad \mathcal{H}_{7}=a_{1}\left(c_{2}+\kappa_{2}\left(\beta_{2}+\theta_{2}\right)\right) .
\end{gathered}
$$

Substituting the solution set (31) into Eqs. (22) and (25), we find that

$$
\pm\left(\xi-\xi_{0}\right)=W \int \frac{d \Psi}{\sqrt{\Lambda(\Psi)}},
$$

where

$$
\Lambda(\Psi)=\Psi^{4}+\frac{\mu_{3}}{\mu_{4}} \Psi^{3}+\frac{\mu_{2}}{\mu_{4}} \Psi^{2}+\frac{\mu_{1}}{\mu_{4}} \Psi+\frac{\mu_{0}}{\mu_{4}}, \quad W=\sqrt{\frac{\chi_{0}}{\mu_{4}}} .
$$

We integrate Eq. (33) and insert the result into Eqs. (27) and (28). Then, we obtain the exact solutions to Eqs. (18) and (19). Consequently, we have the traveling wave solutions to the coupled NLSE with STD and Kerr law nonlinearity as follows:

When $\Lambda(\Psi)=\left(\Psi-\lambda_{1}\right)^{4}$, then we obtain

$$
\begin{aligned}
q(x, t)= & \left\{\tau_{0}+\tau_{1} \lambda_{1} \pm \frac{\tau_{1} W}{B\left(x-\frac{2 a_{1} \kappa_{1}-b_{1} \omega_{1}-\alpha_{1}}{b_{1} \kappa_{1}-1} t\right)-\xi_{0}}\right\} \\
& \left.\times e^{i\left\{-\kappa_{1} x+\left(\frac{\mu_{2} B^{2} \mathcal{H}_{1}^{2}\left(a_{1}-b_{1} v\right)+\chi_{0}\left[\kappa_{1} \mathcal{H}_{5}\left(a_{1} \kappa_{1}-\alpha_{1}\right)+3 \tau_{0}^{2} \mathcal{H}_{3}\left(a_{1}-b_{1} v\right)\right]}{\chi_{0} \mathcal{H}_{5}\left(b_{1} \kappa_{1}-1\right)}\right) t+\sigma_{1}\right.}\right\},
\end{aligned}
$$




$$
\begin{aligned}
r(x, t)= & \left\{\tilde{\tau}_{0}+\tilde{\tau}_{1} \lambda_{1} \pm \frac{\tilde{\tau}_{1} W}{B\left(x-\frac{2 a_{2} \kappa_{2}-b_{2} \omega_{2}-\alpha_{2}}{b_{2} \kappa_{2}-1} t\right)-\xi_{0}}\right\} \\
& \times e^{i\left\{-\kappa_{2} x+\left(\frac{\mu_{2} B^{2}\left(a_{2}-b_{2} v\right)\left(\mathcal{H}_{6}-\mathcal{H}_{7}\right)+\chi_{0}\left[\kappa_{2}\left(\alpha_{2}-a_{2} \kappa_{2}\right)\left(\mathcal{H}_{6}-\mathcal{H}_{7}\right)-3 \tau_{0}^{2} \mathcal{H}_{3}\left(a_{2}-b_{2} v\right)\right]}{\chi_{0} \mathcal{H}_{1}^{2}\left(b_{2} \kappa_{2}-1\right)}\right) t+\sigma_{2}\right\} .}
\end{aligned}
$$

When $\Lambda(\Psi)=\left(\Psi-\lambda_{1}\right)^{3}\left(\Psi-\lambda_{2}\right)$ and $\lambda_{2}>\lambda_{1}$, then we get

$$
\begin{aligned}
q(x, t)= & \left\{\tau_{0}+\tau_{1} \lambda_{1}+\frac{4 W^{2}\left(\lambda_{2}-\lambda_{1}\right) \tau_{1}}{4 W^{2}-\left[\left(\lambda_{1}-\lambda_{2}\right)\left(B\left(x-\frac{2 a_{1} \kappa_{1}-b_{1} \omega_{1}-\alpha_{1}}{b_{1} \kappa_{1}-1} t\right)-\xi_{0}\right)\right]^{2}}\right\} \\
& \times e^{i\left\{-\kappa_{1} x+\left(\frac{\mu_{2} B^{2} \mathcal{H}_{1}^{2}\left(a_{1}-b_{1} v\right)+\chi_{0}\left[\kappa_{1} \mathcal{H}_{5}\left(a_{1} \kappa_{1}-\alpha_{1}\right)+3 \tau_{0}^{2} \mathcal{H}_{3}\left(a_{1}-b_{1} v\right)\right]}{\chi_{0} \mathcal{H}_{5}\left(b_{1} \kappa_{1}-1\right)}\right) t+\sigma_{1}\right\}}, \\
r(x, t)= & \left\{\tilde{\tau}_{0}+\tilde{\tau}_{1} \lambda_{1}+\frac{4 W^{2}\left(\lambda_{2}-\lambda_{1}\right) \tilde{\tau}_{1}}{4 W^{2}-\left[\left(\lambda_{1}-\lambda_{2}\right)\left(B\left(x-\frac{2 a_{2} \kappa_{2}-b_{2} \omega_{2}-\alpha_{2}}{b_{2} \kappa_{2}-1} t\right)-\xi_{0}\right)\right]^{2}}\right\} \\
& \left.\times e^{i\left\{-\kappa_{2} x+\left(\frac{\mu_{2} B^{2}\left(a_{2}-b_{2} v\right)\left(\mathcal{H}_{6}-\mathcal{H}_{7}\right)+\chi_{0}\left[\kappa_{2}\left(\alpha_{2}-a_{2} \kappa_{2}\right)\left(\mathcal{H}_{6}-\mathcal{H}_{7}\right)-3 \tau_{0}^{2} \mathcal{H}_{3}\left(a_{2}-b_{2} v\right)\right]}{\chi_{0} \mathcal{H}_{1}^{2}\left(b_{2} \kappa_{2}-1\right)}\right) t+\sigma_{2}\right.}\right\} .
\end{aligned}
$$

When $\Lambda(\Psi)=\left(\Psi-\lambda_{1}\right)^{2}\left(\Psi-\lambda_{2}\right)^{2}$, then we have

$$
\begin{aligned}
& q(x, t)=\left\{\tau_{0}+\tau_{1} \lambda_{2}+\frac{\left(\lambda_{2}-\lambda_{1}\right) \tau_{1}}{\exp \left[\frac{\lambda_{1}-\lambda_{2}}{W}\left(B\left(x-\frac{2 a_{1} \kappa_{1}-b_{1} \omega_{1}-\alpha_{1}}{b_{1} \kappa_{1}-1} t\right)-\xi_{0}\right)\right]-1}\right\} \\
& \times e^{i\left\{-\kappa_{1} x+\left(\frac{\mu_{2} B^{2} \mathcal{H}_{1}^{2}\left(a_{1}-b_{1} v\right)+\chi_{0}\left[\kappa_{1} \mathcal{H}_{5}\left(a_{1} \kappa_{1}-\alpha_{1}\right)+3 \tau_{0}^{2} \mathcal{H}_{3}\left(a_{1}-b_{1} v\right)\right]}{\chi_{0} \mathcal{H}_{5}\left(b_{1} \kappa_{1}-1\right)}\right) t+\sigma_{1}\right\}}, \\
& r(x, t)=\left\{\tilde{\tau}_{0}+\tilde{\tau}_{1} \lambda_{2}+\frac{\left(\lambda_{2}-\lambda_{1}\right) \tilde{\tau}_{1}}{\exp \left[\frac{\lambda_{1}-\lambda_{2}}{W}\left(B\left(x-\frac{2 a_{2} \kappa_{2}-b_{2} \omega_{2}-\alpha_{2}}{b_{2} \kappa_{2}-1} t\right)-\xi_{0}\right)\right]-1}\right\} \\
& \times e^{i\left\{-\kappa_{2} x+\left(\frac{\mu_{2} B^{2}\left(a_{2}-b_{2} v\right)\left(\mathcal{H}_{6}-\mathcal{H}_{7}\right)+\chi_{0}\left[\kappa_{2}\left(\alpha_{2}-a_{2} \kappa_{2}\right)\left(\mathcal{H}_{6}-\mathcal{H}_{7}\right)-3 \tau_{0}^{2} \mathcal{H}_{3}\left(a_{2}-b_{2} v\right)\right]}{\chi_{0} \mathcal{H}_{1}^{2}\left(b_{2} \kappa_{2}-1\right)}\right) t+\sigma_{2}\right\}},
\end{aligned}
$$

and

$$
\begin{aligned}
& q(x, t)=\left\{\tau_{0}+\tau_{1} \lambda_{1}+\frac{\left(\lambda_{1}-\lambda_{2}\right) \tau_{1}}{\exp \left[\frac{\lambda_{1}-\lambda_{2}}{W}\left(B\left(x-\frac{2 a_{1} \kappa_{1}-b_{1} \omega_{1}-\alpha_{1}}{b_{1} \kappa_{1}-1} t\right)-\xi_{0}\right)\right]-1}\right\} \\
& \times e^{i\left\{-\kappa_{1} x+\left(\frac{\mu_{2} B^{2} \mathcal{H}_{1}^{2}\left(a_{1}-b_{1} v\right)+\chi_{0}\left[\kappa_{1} \mathcal{H}_{5}\left(a_{1} \kappa_{1}-\alpha_{1}\right)+3 \tau_{0}^{2} \mathcal{H}_{3}\left(a_{1}-b_{1} v\right)\right]}{\chi_{0} \mathcal{H}_{5}\left(b_{1} \kappa_{1}-1\right)}\right) t+\sigma_{1}\right\}}, \\
& r(x, t)=\left\{\tilde{\tau}_{0}+\tilde{\tau}_{1} \lambda_{1}+\frac{\left(\lambda_{1}-\lambda_{2}\right) \tilde{\tau}_{1}}{\exp \left[\frac{\lambda_{1}-\lambda_{2}}{W}\left(B\left(x-\frac{2 a_{2} \kappa_{2}-b_{2} \omega_{2}-\alpha_{2}}{b_{2} \kappa_{2}-1} t\right)-\xi_{0}\right)\right]-1}\right\} \\
& \times e^{i\left\{-\kappa_{2} x+\left(\frac{\mu_{2} B^{2}\left(a_{2}-b_{2} v\right)\left(\mathcal{H}_{6}-\mathcal{H}_{7}\right)+\chi_{0}\left[\kappa_{2}\left(\alpha_{2}-a_{2} \kappa_{2}\right)\left(\mathcal{H}_{6}-\mathcal{H}_{7}\right)-3 \tau_{0}^{2} \mathcal{H}_{3}\left(a_{2}-b_{2} v\right)\right]}{\chi_{0} \mathcal{H}_{1}^{2}\left(b_{2} \kappa_{2}-1\right)}\right) t+\sigma_{2}\right\} .}
\end{aligned}
$$


When $\Lambda(\Psi)=\left(\Psi-\lambda_{1}\right)^{2}\left(\Psi-\lambda_{2}\right)\left(\Psi-\lambda_{3}\right)$ and $\lambda_{1}>\lambda_{2}>\lambda_{3}$, then we attain

$$
\begin{aligned}
& q(x, t)=\left\{\tau_{0}+\tau_{1} \lambda_{1}-\frac{2\left(\lambda_{1}-\lambda_{2}\right)\left(\lambda_{1}-\lambda_{3}\right) \tau_{1}}{2 \lambda_{1}-\lambda_{2}-\lambda_{3}+\left(\lambda_{3}-\lambda_{2}\right) \cosh \left(\frac{\sqrt{\left(\lambda_{1}-\lambda_{2}\right)\left(\lambda_{1}-\lambda_{3}\right)}}{W}\left[B\left(x-\frac{2 a_{1} \kappa_{1}-b_{1} \omega_{1}-\alpha_{1}}{b_{1} \kappa_{1}-1} t\right)\right]\right)}\right\} \\
& \times e^{i\left\{-\kappa_{1} x+\left(\frac{\mu_{2} B^{2} \mathcal{H}_{1}^{2}\left(a_{1}-b_{1} v\right)+\chi_{0}\left[\kappa_{1} \mathcal{H}_{5}\left(a_{1} \kappa_{1}-\alpha_{1}\right)+3 \tau_{0}^{2} \mathcal{H}_{3}\left(a_{1}-b_{1} v\right)\right]}{\chi_{0} \mathcal{H}_{5}\left(b_{1} \kappa_{1}-1\right)}\right) t+\sigma_{1}\right\}}, \\
& r(x, t)=\left\{\tilde{\tau}_{0}+\tilde{\tau}_{1} \lambda_{1}-\frac{2\left(\lambda_{1}-\lambda_{2}\right)\left(\lambda_{1}-\lambda_{3}\right) \tilde{\tau}_{1}}{2 \lambda_{1}-\lambda_{2}-\lambda_{3}+\left(\lambda_{3}-\lambda_{2}\right) \cosh \left(\frac{\sqrt{\left(\lambda_{1}-\lambda_{2}\right)\left(\lambda_{1}-\lambda_{3}\right)}}{W}\left[B\left(x-\frac{2 a_{2} \kappa_{2}-b_{2} \omega_{2}-\alpha_{2}}{b_{2} \kappa_{2}-1} t\right)\right]\right)}\right\} \\
& \times e^{i\left\{-\kappa_{2} x+\left(\frac{\mu_{2} B^{2}\left(a_{2}-b_{2} v\right)\left(\mathcal{H}_{6}-\mathcal{H}_{7}\right)+\chi_{0}\left[\kappa_{2}\left(\alpha_{2}-a_{2} \kappa_{2}\right)\left(\mathcal{H}_{6}-\mathcal{H}_{7}\right)-3 \tau_{0}^{2} \mathcal{H}_{3}\left(a_{2}-b_{2} v\right)\right]}{\chi_{0} \mathcal{H}_{1}^{2}\left(b_{2} \kappa_{2}-1\right)}\right) t+\sigma_{2}\right\}} .
\end{aligned}
$$

When $\Lambda(\Psi)=\left(\Psi-\lambda_{1}\right)\left(\Psi-\lambda_{2}\right)\left(\Psi-\lambda_{3}\right)\left(\Psi-\lambda_{4}\right)$ and $\lambda_{1}>\lambda_{2}>\lambda_{3}>\lambda_{4}$, then we achieve

$$
\begin{aligned}
& q(x, t)=\left\{\tau_{0}+\tau_{1} \lambda_{2}+\frac{\tau_{1}\left(\lambda_{1}-\lambda_{2}\right)\left(\lambda_{4}-\lambda_{2}\right)}{\lambda_{4}-\lambda_{2}+\left(\lambda_{1}-\lambda_{4}\right) \operatorname{sn}^{2}\left[ \pm \frac{\sqrt{\left(\lambda_{1}-\lambda_{3}\right)\left(\lambda_{2}-\lambda_{4}\right)}}{2 W}\left[B\left(x-\frac{2 a_{1} \kappa_{1}-b_{1} \omega_{1}-\alpha_{1}}{b_{1} \kappa_{1}-1} t\right)-\xi_{0}\right], l\right]}\right\} \\
& \times e^{i\left\{-\kappa_{1} x+\left(\frac{\mu_{2} B^{2} \mathcal{H}_{1}^{2}\left(a_{1}-b_{1} v\right)+\chi_{0}\left[\kappa_{1} \mathcal{H}_{5}\left(a_{1} \kappa_{1}-\alpha_{1}\right)+3 \tau_{0}^{2} \mathcal{H}_{3}\left(a_{1}-b_{1} v\right)\right]}{\chi_{0} \mathcal{H}_{5}\left(b_{1} \kappa_{1}-1\right)}\right) t+\sigma_{1}\right\}}, \\
& r(x, t)=\left\{\tilde{\tau}_{0}+\tilde{\tau}_{1} \lambda_{2}+\frac{\tilde{\tau}_{1}\left(\lambda_{1}-\lambda_{2}\right)\left(\lambda_{4}-\lambda_{2}\right)}{\lambda_{4}-\lambda_{2}+\left(\lambda_{1}-\lambda_{4}\right) \mathrm{sn}^{2}\left[ \pm \frac{\sqrt{\left(\lambda_{1}-\lambda_{3}\right)\left(\lambda_{2}-\lambda_{4}\right)}}{2 W}\left[B\left(x-\frac{2 a_{2} \kappa_{2}-b_{2} \omega_{2}-\alpha_{2}}{b_{2} \kappa_{2}-1} t\right)-\xi_{0}\right], l\right]}\right\} \\
& \times e^{i\left\{-\kappa_{2} x+\left(\frac{\mu_{2} B^{2}\left(a_{2}-b_{2} v\right)\left(\mathcal{H}_{6}-\mathcal{H}_{7}\right)+\chi_{0}\left[\kappa_{2}\left(\alpha_{2}-a_{2} \kappa_{2}\right)\left(\mathcal{H}_{6}-\mathcal{H}_{7}\right)-3 \tau_{0}^{2} \mathcal{H}_{3}\left(a_{2}-b_{2} v\right)\right]}{\chi_{0} \mathcal{H}_{1}^{2}\left(b_{2} \kappa_{2}-1\right)}\right) t+\sigma_{2}\right\}},
\end{aligned}
$$

where

$$
l^{2}=\frac{\left(\lambda_{2}-\lambda_{3}\right)\left(\lambda_{1}-\lambda_{4}\right)}{\left(\lambda_{1}-\lambda_{3}\right)\left(\lambda_{2}-\lambda_{4}\right)}
$$

Also, $\lambda_{i}(i=1, \ldots, 4)$ are the roots of the polynomial equation

$$
\Lambda(\Psi)=0 .
$$

When $\tau_{0}=-\tau_{1} \lambda_{1}, \tilde{\tau}_{0}=-\tilde{\tau}_{1} \lambda_{1}$ and $\xi_{0}=0$, then we can reduce the solutions (35)-(44) to plane wave solutions

$$
\begin{gathered}
q(x, t)=\left\{ \pm \frac{\tau_{1} W}{B\left(x-\frac{2 a_{1} \kappa_{1}-b_{1} \omega_{1}-\alpha_{1}}{b_{1} \kappa_{1}-1} t\right)}\right\} e^{i\left\{-\kappa_{1} x+\left(\frac{\mu_{2} B^{2} \mathcal{H}_{1}^{2}\left(a_{1}-b_{1} v\right)+\chi_{0}\left[\kappa_{1} \mathcal{H}_{5}\left(a_{1} \kappa_{1}-\alpha_{1}\right)+3 \tau_{0}^{2} \mathcal{H}_{3}\left(a_{1}-b_{1} v\right)\right]}{\chi_{0} \mathcal{H}_{5}\left(b_{1} \kappa_{1}-1\right)}\right) t+\sigma_{1}\right\}}, \\
r(x, t)=\left\{ \pm \frac{\tilde{\tau}_{1} W}{B\left(x-\frac{2 a_{2} \kappa_{2}-b_{2} \omega_{2}-\alpha_{2}}{b_{2} \kappa_{2}-1} t\right)}\right\} e^{i\left\{-\kappa_{2} x+\left(\frac{\mu_{2} B^{2}\left(a_{2}-b_{2} v\right)\left(\mathcal{H}_{6}-\mathcal{H}_{7}\right)+\chi_{0}\left[\kappa_{2}\left(\alpha_{2}-a_{2} \kappa_{2}\right)\left(\mathcal{H}_{6}-\mathcal{H}_{7}\right)-3 \tau_{0}^{2} \mathcal{H}_{3}\left(a_{2}-b_{2} v\right)\right]}{\chi_{0} \mathcal{H}_{1}^{2}\left(b_{2} \kappa_{2}-1\right)}\right) t+\sigma_{2}\right\}},
\end{gathered}
$$




$$
\begin{aligned}
& q(x, t)=\left\{\frac{4 W^{2}\left(\lambda_{2}-\lambda_{1}\right) \tau_{1}}{4 W^{2}-\left[\left(\lambda_{1}-\lambda_{2}\right)\left(B\left(x-\frac{2 a_{1} \kappa_{1}-b_{1} \omega_{1}-\alpha_{1}}{b_{1} \kappa_{1}-1} t\right)\right)\right]^{2}}\right\} \\
& \times e^{i\left\{-\kappa_{1} x+\left(\frac{\mu_{2} B^{2} \mathcal{H}_{1}^{2}\left(a_{1}-b_{1} v\right)+\chi_{0}\left[\kappa_{1} \mathcal{H}_{5}\left(a_{1} \kappa_{1}-\alpha_{1}\right)+3 \tau_{0}^{2} \mathcal{H}_{3}\left(a_{1}-b_{1} v\right)\right]}{\chi_{0} \mathcal{H}_{5}\left(b_{1} \kappa_{1}-1\right)}\right) t+\sigma_{1}\right\}}, \\
& r(x, t)=\left\{\frac{4 W^{2}\left(\lambda_{2}-\lambda_{1}\right) \tilde{\tau}_{1}}{4 W^{2}-\left[\left(\lambda_{1}-\lambda_{2}\right)\left(B\left(x-\frac{2 a_{2} \kappa_{2}-b_{2} \omega_{2}-\alpha_{2}}{b_{2} \kappa_{2}-1} t\right)\right]^{2}\right.}\right\} \\
& \times e^{i\left\{-\kappa_{2} x+\left(\frac{\mu_{2} B^{2}\left(a_{2}-b_{2} v\right)\left(\mathcal{H}_{6}-\mathcal{H}_{7}\right)+\chi_{0}\left[\kappa_{2}\left(\alpha_{2}-a_{2} \kappa_{2}\right)\left(\mathcal{H}_{6}-\mathcal{H}_{7}\right)-3 \tau_{0}^{2} \mathcal{H}_{3}\left(a_{2}-b_{2} v\right)\right]}{\chi_{0} \mathcal{H}_{1}^{2}\left(b_{2} \kappa_{2}-1\right)}\right) t+\sigma_{2}\right\}},
\end{aligned}
$$

singular soliton solutions

$$
\begin{aligned}
q(x, t)= & \frac{\left(\lambda_{2}-\lambda_{1}\right) \tau_{1}}{2}\left\{1 \mp \operatorname{coth}\left[\frac{\lambda_{1}-\lambda_{2}}{2 W}\left(B\left(x-\frac{2 a_{1} \kappa_{1}-b_{1} \omega_{1}-\alpha_{1}}{b_{1} \kappa_{1}-1} t\right)\right)\right]\right\} \\
& \times e^{i\left\{-\kappa_{1} x+\left(\frac{\mu_{2} B^{2} \mathcal{H}_{1}^{2}\left(a_{1}-b_{1} v\right)+\chi_{0}\left[\kappa_{1} \mathcal{H}_{5}\left(a_{1} \kappa_{1}-\alpha_{1}\right)+3 \tau_{0}^{2} \mathcal{H}_{3}\left(a_{1}-b_{1} v\right)\right]}{\chi_{0} \mathcal{H}_{5}\left(b_{1} \kappa_{1}-1\right)}\right) t+\sigma_{1}\right\}}, \\
r(x, t)= & \frac{\left(\lambda_{2}-\lambda_{1}\right) \tilde{\tau}_{1}}{2}\left\{1 \mp \operatorname{coth}\left[\frac{\lambda_{1}-\lambda_{2}}{2 W}\left(B\left(x-\frac{2 a_{2} \kappa_{2}-b_{2} \omega_{2}-\alpha_{2}}{b_{2} \kappa_{2}-1} t\right)\right)\right]\right\} \\
& \times e^{i\left\{-\kappa_{2} x+\left(\frac{\mu_{2} B^{2}\left(a_{2}-b_{2} v\right)\left(\mathcal{H}_{6}-\mathcal{H}_{7}\right)+\chi_{0}\left[\kappa_{2}\left(\alpha_{2}-a_{2} \kappa_{2}\right)\left(\mathcal{H}_{6}-\mathcal{H}_{7}\right)-3 \tau_{0}^{2} \mathcal{H}_{3}\left(a_{2}-b_{2} v\right)\right]}{\chi_{0} \mathcal{H}_{1}^{2}\left(b_{2} \kappa_{2}-1\right)}\right) t+\sigma_{2}\right\}},
\end{aligned}
$$

and bright soliton solutions

$$
\begin{aligned}
q(x, t)= & \left\{\frac{A}{C+\cosh \left[D\left(B\left(x-\frac{2 a \kappa_{1} \kappa_{1}-b_{1} \omega_{1}-\alpha_{1}}{b_{1} \kappa_{1}-1} t\right)\right)\right]}\right\} \\
& \times e^{i\left\{-\kappa_{1} x+\left(\frac{\mu_{2} B^{2} \mathcal{H}_{1}^{2}\left(a_{1}-b_{1} v\right)+\chi_{0}\left[\kappa_{1} \mathcal{H}_{5}\left(a_{1} \kappa_{1}-\alpha_{1}\right)+3 \tau_{0}^{2} \mathcal{H}_{3}\left(a_{1}-b_{1} v\right)\right]}{\chi_{0} \mathcal{H}_{5}\left(b_{1} \kappa_{1}-1\right)}\right) t+\sigma_{1}\right\}}, \\
r(x, t)= & \left\{\frac{\tilde{A}}{C+\cosh \left[D\left(B\left(x-\frac{2 a_{2} \kappa_{2}-b_{2} \omega_{2}-\alpha_{2}}{b_{2} \kappa_{2}-1} t\right)\right)\right]}\right\} \\
\times & e^{i\left\{-\kappa_{2} x+\left(\frac{\mu_{2} B^{2}\left(a_{2}-b_{2} v\right)\left(\mathcal{H}_{6}-\mathcal{H}_{7}\right)+\chi_{0}\left[\kappa_{2}\left(\alpha_{2}-a_{2} \kappa_{2}\right)\left(\mathcal{H}_{6}-\mathcal{H}_{7}\right)-3 \tau_{0}^{2} \mathcal{H}_{3}\left(a_{2}-b_{2} v\right)\right]}{\chi_{0} \mathcal{H}_{1}^{2}\left(b_{2} \kappa_{2}-1\right)}\right) t+\sigma_{2}\right\},},
\end{aligned}
$$

where

$$
A=\frac{2\left(\lambda_{1}-\lambda_{2}\right)\left(\lambda_{1}-\lambda_{3}\right) \tau_{1}}{\lambda_{3}-\lambda_{2}}, \quad \tilde{A}=\frac{2\left(\lambda_{1}-\lambda_{2}\right)\left(\lambda_{1}-\lambda_{3}\right) \tilde{\tau}_{1}}{\lambda_{3}-\lambda_{2}}, \quad D=\frac{\sqrt{\left(\lambda_{1}-\lambda_{2}\right)\left(\lambda_{1}-\lambda_{3}\right)}}{W}, \quad C=\frac{2 \lambda_{1}-\lambda_{2}-\lambda_{3}}{\lambda_{3}-\lambda_{2}} .
$$

Here, $A$ and $\tilde{A}$ are the amplitudes of the solitons, while $D$ is related to inverse width of the solitons. These solitons exist for $\tau_{1}<0$ and $\tilde{\tau}_{1}<0$. Moreover, when $\tau_{0}=-\tau_{1} \lambda_{2}, \tilde{\tau}_{0}=-\tilde{\tau}_{1} \lambda_{2}$ and $\xi_{0}=0$, we can write the Jacobi elliptic 
function solutions (45) and (46) as follows:

$$
\begin{aligned}
q(x, t)= & \left\{\frac{A_{1}}{C_{1}+\mathrm{sn}^{2}\left[D_{j}\left[B\left(x-\frac{2 a_{1} \kappa_{1}-b_{1} \omega_{1}-\alpha_{1}}{b_{1} \kappa_{1}-1} t\right)\right], \frac{\left(\lambda_{2}-\lambda_{3}\right)\left(\lambda_{1}-\lambda_{4}\right)}{\left(\lambda_{1}-\lambda_{3}\right)\left(\lambda_{2}-\lambda_{4}\right)}\right\}}\right\} \\
r(x, t)= & \times \frac{\tilde{A}_{1}}{e^{i\left\{-\kappa_{1} x+\left(\frac{\mu_{2} B^{2} \mathcal{H}_{1}^{2}\left(a_{1}-b_{1} v\right)+\chi_{0}\left[\kappa_{1} \mathcal{H}_{5}\left(a_{1} \kappa_{1}-\alpha_{1}\right)+3 \tau_{0}^{2} \mathcal{H}_{3}\left(a_{1}-b_{1} v\right)\right]}{\chi_{0} \mathcal{H}_{5}\left(b_{1} \kappa_{1}-1\right)}\right) t+\sigma_{1}\right\}},} \\
& \times e^{i\left\{-\kappa_{2} x+\left(\frac{\mu_{2} B^{2}\left(a_{2}-b_{2} v\right)\left(\mathcal{H}_{6}-\mathcal{H}_{7}\right)+\chi_{0}\left[\kappa_{2}\left(\alpha_{2}-a_{2} \kappa_{2}\right)\left(\mathcal{H}_{6}-\mathcal{H}_{7}\right)-3 \tau_{0}^{2} \mathcal{H}_{3}\left(a_{2}-b_{2} v\right)\right]}{\chi_{0} \mathcal{H}_{1}^{2}\left(b_{2} \kappa_{2}-1\right)}\right) t+\sigma_{2}\right\}},
\end{aligned}
$$

where

$$
\begin{gathered}
A_{1}=\frac{\tau_{1}\left(\lambda_{1}-\lambda_{2}\right)\left(\lambda_{4}-\lambda_{2}\right)}{\lambda_{1}-\lambda_{4}}, \quad \tilde{A}_{1}=\frac{\tilde{\tau}_{1}\left(\lambda_{1}-\lambda_{2}\right)\left(\lambda_{4}-\lambda_{2}\right)}{\lambda_{1}-\lambda_{4}}, \\
C_{1}=\frac{\lambda_{4}-\lambda_{2}}{\lambda_{1}-\lambda_{4}}, \quad D_{j}=\frac{(-1)^{j} \sqrt{\left(\lambda_{1}-\lambda_{3}\right)\left(\lambda_{2}-\lambda_{4}\right)}}{2 W}, \quad(j=1,2) .
\end{gathered}
$$

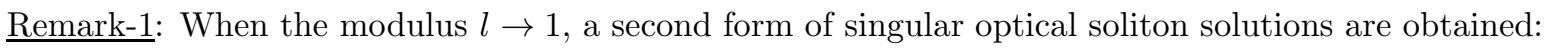

$$
\begin{aligned}
& q(x, t)=\left\{\frac{A_{1}}{C_{1}+\tanh ^{2}\left[D_{j}\left(B\left(x-\frac{2 a_{1} \kappa_{1}-b_{1} \omega_{1}-\alpha_{1}}{b_{1} \kappa_{1}-1} t\right)\right)\right]}\right\} \\
& \times e^{i\left\{-\kappa_{1} x+\left(\frac{\mu_{2} B^{2} \mathcal{H}_{1}^{2}\left(a_{1}-b_{1} v\right)+\chi_{0}\left[\kappa_{1} \mathcal{H}_{5}\left(a_{1} \kappa_{1}-\alpha_{1}\right)+3 \tau_{0}^{2} \mathcal{H}_{3}\left(a_{1}-b_{1} v\right)\right]}{\chi_{0} \mathcal{H}_{5}\left(b_{1} \kappa_{1}-1\right)}\right) t+\sigma_{1}\right\}}, \\
& r(x, t)=\left\{\frac{\tilde{A}_{1}}{C_{1}+\tanh ^{2}\left[D_{j}\left(B\left(x-\frac{2 a_{2} \kappa_{2}-b_{2} \omega_{2}-\alpha_{2}}{b_{2} \kappa_{2}-1} t\right)\right)\right]}\right\} \\
& \times e^{i\left\{-\kappa_{2} x+\left(\frac{\mu_{2} B^{2}\left(a_{2}-b_{2} v\right)\left(\mathcal{H}_{6}-\mathcal{H}_{7}\right)+\chi_{0}\left[\kappa_{2}\left(\alpha_{2}-a_{2} \kappa_{2}\right)\left(\mathcal{H}_{6}-\mathcal{H}_{7}\right)-3 \tau_{0}^{2} \mathcal{H}_{3}\left(a_{2}-b_{2} v\right)\right]}{\chi_{0} \mathcal{H}_{1}^{2}\left(b_{2} \kappa_{2}-1\right)}\right) t+\sigma_{2}\right\},},
\end{aligned}
$$

where $\lambda_{3}=\lambda_{4}$.

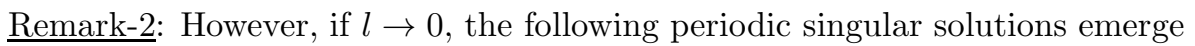

$$
\begin{aligned}
& q(x, t)=\left\{\frac{A_{1}}{C_{1}+\sin ^{2}\left[D_{j}\left(B\left(x-\frac{2 a_{1} \kappa_{1}-b_{1} \omega_{1}-\alpha_{1}}{b_{1} \kappa_{1}-1}\right)\right)\right]}\right\} \\
& r(x, t)=\left.\times \frac{e^{i\left\{-\kappa_{1} x+\left(\frac{\mu_{2} B^{2} \mathcal{H}_{1}^{2}\left(a_{1}-b_{1} v\right)+\chi_{0}\left[\kappa_{1} \mathcal{H}_{5}\left(a_{1} \kappa_{1}-\alpha_{1}\right)+3 \tau_{0}^{2} \mathcal{H}_{3}\left(a_{1}-b_{1} v\right)\right]}{\chi_{0} \mathcal{H}_{5}\left(b_{1} \kappa_{1}-1\right)}\right) t+\sigma_{1}\right\}},}{C_{1}+\sin ^{2}\left[D_{j}\left(B\left(x-\frac{2 a_{2} \kappa_{2}-b_{2} \omega_{2}-\alpha_{2}}{b_{2} \kappa_{2}-1} t\right)\right)\right]}\right\} \\
& \times e^{i\left\{-\kappa_{2} x+\left(\frac{\mu_{2} B^{2}\left(a_{2}-b_{2} v\right)\left(\mathcal{H}_{6}-\mathcal{H}_{7}\right)+\chi_{0}\left[\kappa_{2}\left(\alpha_{2}-a_{2} \kappa_{2}\right)\left(\mathcal{H}_{6}-\mathcal{H}_{7}\right)-3 \tau_{0}^{2} \mathcal{H}_{3}\left(a_{2}-b_{2} v\right)\right]}{\chi_{0} \mathcal{H}_{1}^{2}\left(b_{2} \kappa_{2}-1\right)}\right) t+\sigma_{2}\right\},}
\end{aligned}
$$

where $\lambda_{2}=\lambda_{3}$. 


\section{PARABOLIC LAW NONLINEARITY}

For parabolic law nonlinearity, the coupled NLSE in birefringent fibers with GVD and STD is given by [1]

$$
\begin{aligned}
& i q_{t}+a_{1} q_{x x}+b_{1} q_{x t}+\left(c_{1}|q|^{2}+d_{1}|r|^{2}\right) q+\left(\delta_{1}|q|^{4}+\eta_{1}|q|^{2}|r|^{2}+\zeta_{1}|r|^{4}\right) q \\
+ & i\left\{\alpha_{1} q_{x}+\beta_{1}\left(|q|^{2} q\right)_{x}+\nu_{1}\left(|q|^{2}\right)_{x} q+\theta_{1}|q|^{2} q_{x}+\gamma_{1} q_{x x x}\right\}=0 \\
& i r_{t}+a_{2} r_{x x}+b_{2} r_{x t}+\left(c_{2}|r|^{2}+d_{2}|q|^{2}\right) r+\left(\delta_{2}|r|^{4}+\eta_{2}|r|^{2}|q|^{2}+\zeta_{2}|q|^{4}\right) q \\
+ & i\left\{\alpha_{2} r_{x}+\beta_{2}\left(|r|^{2} r\right)_{x}+\nu_{2}\left(|r|^{2}\right)_{x} r+\theta_{2}|r|^{2} r_{x}+\gamma_{2} r_{x x x}\right\}=0 .
\end{aligned}
$$

In (65) and (66), terms with $c_{l}$ and $\delta_{l}$ are asociated with SPM while $d_{l}, \eta_{l}$ and $\zeta_{l}$ are from XPM for parabolic law nonlinearity. The rest of the terms have the same interpretation as with the Kerr law nonlinearity. Substituting the same hypothesis as in (3) and (4) into (65) and (66), and decomposing into real and imaginary parts yields

$$
\begin{gathered}
\left(\omega_{l}+a_{l} \kappa_{l}^{2}-b_{l} \kappa_{l} \omega_{l}-\alpha_{l} \kappa_{l}+\gamma_{l} \kappa_{l}^{3}\right) P_{l}-d_{l} P_{l} P_{l}^{2}-\delta_{l} P_{l}^{5}-\eta_{l} P_{l}^{3} P_{\bar{l}}-\zeta_{l} P_{l} P_{\bar{l}}^{4}-\left(\beta_{l} \kappa_{l}+\theta_{l} \kappa_{l}+c_{l}\right) P_{l}^{3}-\left(a_{l}+3 \gamma_{l} \kappa_{l}\right) \frac{\partial^{2} P_{l}}{\partial x^{2}}-b_{l} \frac{\partial^{2} P_{l}}{\partial x \partial t}=0 \\
\left(1-b_{l} \kappa_{l}\right) \frac{\partial P_{l}}{\partial t}-\left(2 a_{l} \kappa_{l}-b_{l} \omega_{l}-\alpha_{l}+3 \gamma_{l} \kappa_{l}^{2}\right) \frac{\partial P_{l}}{\partial x}+\left(3 \beta_{l}+2 \nu_{l}+\theta_{l}\right) P_{l}^{2} \frac{\partial P_{l}}{\partial x}+\gamma_{l} \frac{\partial^{3} P_{l}}{\partial x^{3}}=0
\end{gathered}
$$

for $l=1,2$ and $\bar{l}=3-l$. With travelling wave transformation (8), we have

$$
\begin{gathered}
\left(\omega_{l}+a_{l} \kappa_{l}^{2}-b_{l} \kappa_{l} \omega_{l}-\alpha_{l} \kappa_{l}+\gamma_{l} \kappa_{l}^{3}\right) U_{l}-d_{l} U_{l} U_{\bar{l}}^{2}-\delta_{l} U_{l}^{5}-\eta_{l} U_{l}^{3} U_{\bar{l}}-\zeta_{l} U_{l} U_{l}^{4}-\left(\beta_{l} \kappa_{l}+\theta_{l} \kappa_{l}+c_{l}\right) U_{l}^{3}-\left(a_{l}-b_{l} v+3 \gamma_{l} \kappa_{l}\right) B^{2} \frac{d^{2} U_{l}}{d \xi^{2}}=0 \\
-\left(v\left(1-b_{l} \kappa_{l}\right)+2 a_{l} \kappa_{l}-b_{l} \omega_{l}-\alpha_{l}+3 \gamma_{l} \kappa_{l}^{2}\right) B \frac{d U_{l}}{d \xi}+\left(3 \beta_{l}+2 \nu_{l}+\theta_{l}\right) B U_{l}^{2} \frac{d U_{l}}{d \xi}+\gamma_{l} B^{3} \frac{d^{3} U_{l}}{d \xi^{3}}=0
\end{gathered}
$$

From (70), once again $\gamma_{l}=0$. The speed $v$ of the soliton from the linearly independent functions is also given by (14). The constraint conditions (15) and (16) also fall out from these linearly independent functions. Consequently, the real part Eq. (69) changes to

$$
\begin{aligned}
& \left(\omega_{1}+a_{1} \kappa_{1}^{2}-b_{1} \kappa_{1} \omega_{1}-\alpha_{1} \kappa_{1}\right) U_{1}-d_{1} U_{1} U_{2}^{2}-\delta_{1} U_{1}^{5}-\eta_{1} U_{1}^{3} U_{2}-\zeta_{1} U_{1} U_{2}^{4}-\left(\beta_{1} \kappa_{1}+\theta_{1} \kappa_{1}+c_{1}\right) U_{1}^{3}-\left(a_{1}-b_{1} v\right) B^{2} \frac{d^{2} U_{1}}{d \xi^{2}}=0 \\
& \left(\omega_{2}+a_{2} \kappa_{2}^{2}-b_{2} \kappa_{2} \omega_{2}-\alpha_{2} \kappa_{2}\right) U_{2}-d_{2} U_{2} U_{1}^{2}-\delta_{2} U_{2}^{5}-\eta_{2} U_{2}^{3} U_{1}-\zeta_{2} U_{2} U_{1}^{4}-\left(\beta_{2} \kappa_{2}+\theta_{2} \kappa_{2}+c_{2}\right) U_{2}^{3}-\left(a_{2}-b_{2} v\right) B^{2} \frac{d^{2} U_{2}}{d \xi^{2}}=0
\end{aligned}
$$

We will now analyze the system of equations (71) and (72) to obtain soliton solutions by the extended trial equation method in the next subsection.

\subsection{EXTENDED TRIAL EQUATION METHOD}

Balancing $U_{l}^{\prime \prime}$ with $U_{l}^{5}$ in Eqs. (71) and (72), we find that

$$
N+2=5 N \Leftrightarrow 2=4 N \Leftrightarrow N=\frac{1}{2} .
$$

When $\eta_{l}=0$, in order to obtain an analytic solution we use the transformation

$$
U_{1}=V_{1}^{\frac{1}{2}}=V_{2}^{\frac{1}{2}}=U_{2}
$$


that will reduce Eqs. (71) and (72) into the system of equations

$$
\begin{aligned}
& 4\left(\omega_{1}+a_{1} \kappa_{1}^{2}-b_{1} \kappa_{1} \omega_{1}-\alpha_{1} \kappa_{1}\right) V_{1}^{2}-4\left(d_{1}+\beta_{1} \kappa_{1}+\theta_{1} \kappa_{1}+c_{1}\right) V_{1}^{3}-4\left(\delta_{1}+\zeta_{1}\right) V_{1}^{4}+\left(a_{1}-b_{1} v\right) B^{2}\left\{\left(V_{1}^{\prime}\right)^{2}-2 V_{1} V_{1}^{\prime \prime}\right\}=0 \\
& 4\left(\omega_{2}+a_{2} \kappa_{2}^{2}-b_{2} \kappa_{2} \omega_{2}-\alpha_{2} \kappa_{2}\right) V_{2}^{2}-4\left(d_{2}+\beta_{2} \kappa_{2}+\theta_{2} \kappa_{2}+c_{2}\right) V_{2}^{3}-4\left(\delta_{2}+\zeta_{2}\right) V_{2}^{4}+\left(a_{2}-b_{2} v\right) B^{2}\left\{\left(V_{2}^{\prime}\right)^{2}-2 V_{2} V_{2}^{\prime \prime}\right\}=0 .
\end{aligned}
$$

We substitute Eqs. (20), (21), (23) and (24) into Eqs. (75) and (76). Then, we balance the order of $V_{l} V_{l}^{\prime \prime}$ and $V_{l}^{4}$ in Eqs. (75) and (76) and find that

$$
\varsigma=\tilde{\varsigma}=\frac{\epsilon-\rho-2}{2} .
$$

When $\epsilon=4, \rho=0$ and $\varsigma=\tilde{\varsigma}=1$ in Eq. (77), Eqs. (75) and (76) have the solutions in the form

$$
\begin{aligned}
& V_{1}(\xi)=\tau_{0}+\tau_{1} \Psi, \\
& V_{2}(\xi)=\tilde{\tau}_{0}+\tilde{\tau}_{1} \Psi,
\end{aligned}
$$

where $\tau_{i}, \tilde{\tau}_{i}(\mathrm{i}=0,1)$ are constants to be determined later. We substitute Eqs. (78)-(79) into Eqs. (75)-(76) and collect the coefficients of $\Psi$. Then, we solve the resulting system and find the following results:

$$
\begin{aligned}
& \mu_{0}=\frac{\tau_{0} \tilde{\tau}_{0}\left(-3 \tau_{1}^{3} \chi_{0} \tilde{\tau}_{0}^{2} \mathcal{H}_{3}\left(a_{2}-b_{2} v\right)+3 \tau_{0}^{2} \chi_{0} \tilde{\tau}_{1}^{3} \mathcal{H}_{4}\left(a_{1}-b_{1} v\right)+\tau_{1}^{2} \tilde{\tau}_{0} \tilde{\tau}_{1} \mathcal{H}_{1}+\tau_{0} \tau_{1} \tilde{\tau}_{1}^{2} \mathcal{H}_{2}\right)}{2 \tau_{1}^{2} \tilde{\tau}_{1}^{2} B^{2}\left(\tau_{1} \tilde{\tau}_{0}-\tau_{0} \tilde{\tau}_{1}\right)\left(a_{1}-b_{1} v\right)\left(b_{2} v-a_{2}\right)}, \\
& \mu_{1}=\frac{\chi_{0}\left(\tilde{\tau}_{1} \mathcal{H}_{4} \mathcal{H}_{6}\left(a_{1}-b_{1} v\right)+\tau_{1} \mathcal{H}_{3} \mathcal{H}_{5}\left(b_{2} v-a_{2}\right)\right)+2 \mu_{2} \tau_{1} \tilde{\tau}_{1} B^{2}\left(\tau_{1}^{2} \tilde{\tau}_{0}^{2}-\tau_{0}^{2} \tilde{\tau}_{1}^{2}\right)\left(a_{1}-b_{1} v\right)\left(b_{2} v-a_{2}\right)}{2 \tau_{1}^{2} \tilde{\tau}_{1}^{2} B^{2}\left(\tau_{1} \tilde{\tau}_{0}-\tau_{0} \tilde{\tau}_{1}\right)\left(a_{1}-b_{1} v\right)\left(b_{2} v-a_{2}\right)}, \\
& \mu_{3}=\frac{2 \chi_{0}\left(\tau_{1}^{2} \tilde{\tau}_{0} \mathcal{H}_{3}\left(a_{2}-b_{2} v\right)+\tau_{0} \tilde{\tau}_{1}^{2} \mathcal{H}_{4}\left(b_{1} v-a_{1}\right)\right)}{B^{2}\left(\tau_{1} \tilde{\tau}_{0}-\tau_{0} \tilde{\tau}_{1}\right)\left(a_{1}-b_{1} v\right)\left(b_{2} v-a_{2}\right)}, \quad \mu_{4}=\frac{\tau_{1} \chi_{0} \tilde{\tau}_{1}\left(\tilde{\tau}_{1} \mathcal{H}_{4}\left(b_{1} v-a_{1}\right)+\tau_{1} \mathcal{H}_{3}\left(a_{2}-b_{2} v\right)\right)}{2 B^{2}\left(\tau_{1} \tilde{\tau}_{0}-\tau_{0} \tilde{\tau}_{1}\right)\left(a_{1}-b_{1} v\right)\left(b_{2} v-a_{2}\right)}, \\
& \omega_{1}=\frac{a_{1}\left(\mathcal{H}_{9}-\tau_{1}^{2} \tilde{\tau}_{0}\left(a_{2}-b_{2} v\right)\left(\mu_{2} B^{2}-4 \kappa_{1}^{2} \chi_{0}\right)\right)+3 \tau_{0}^{2} \chi_{0} \tilde{\tau}_{1}^{2} \mathcal{H}_{4}\left(a_{1}-b_{1} v\right)+\mathcal{H}_{7}+\mathcal{H}_{8}}{4 \tau_{1} \chi_{0}\left(\tau_{1} \tilde{\tau}_{0}-\tau_{0} \tilde{\tau}_{1}\right)\left(b_{1} \kappa_{1}-1\right)\left(a_{2}-b_{2} v\right)}, \\
& \omega_{2}=\frac{3 \tau_{1}^{2} \chi_{0} \tilde{\tau}_{0}^{2} \mathcal{H}_{3}\left(a_{2}-b_{2} v\right)+\mathcal{H}_{10}-\mathcal{H}_{11}}{4 \chi_{0} \tilde{\tau}_{1}\left(\tau_{0} \tilde{\tau}_{1}-\tau_{1} \tilde{\tau}_{0}\right)\left(b_{2} \kappa_{2}-1\right)\left(a_{1}-b_{1} v\right)}, \\
& \delta_{1}=\frac{-8 \zeta_{1} \tau_{1}^{2} \tilde{\tau}_{0}\left(a_{2}-b_{2} v\right)+\tau_{1} \tilde{\tau}_{1}\left(a_{2}-b_{2} v\right)\left(8 \zeta_{1} \tau_{0}+3 \mathcal{H}_{3}\right)-3 \tilde{\tau}_{1}^{2} \mathcal{H}_{4}\left(a_{1}-b_{1} v\right)}{8 \tau_{1}\left(\tau_{1} \tilde{\tau}_{0}-\tau_{0} \tilde{\tau}_{1}\right)\left(a_{2}-b_{2} v\right)}, \\
& \delta_{2}=\frac{\tilde{\tau}_{1}\left(a_{1}-b_{1} v\right)\left(\tau_{1}\left(8 \zeta_{2} \tilde{\tau}_{0}+3 \mathcal{H}_{4}\right)-8 \zeta_{2} \tau_{0} \tilde{\tau}_{1}\right)-3 a_{2} \tau_{1}^{2} \mathcal{H}_{3}+3 b_{2} \tau_{1}^{2} v \mathcal{H}_{3}}{8 \tilde{\tau}_{1}\left(\tau_{0} \tilde{\tau}_{1}-\tau_{1} \tilde{\tau}_{0}\right)\left(a_{1}-b_{1} v\right)}, \\
& \mu_{2}=\mu_{2}, \quad \chi_{0}=\chi_{0}, \quad \tau_{0}=\tau_{0}, \quad \tau_{1}=\tau_{1}, \quad \tilde{\tau}_{0}=\tilde{\tau}_{0}, \quad \tilde{\tau}_{1}=\tilde{\tau}_{1},
\end{aligned}
$$

where

$$
\begin{gathered}
\mathcal{H}_{1}=\left(a_{2}-b_{2} v\right)\left(-2 \mu_{2} B^{2}\left(a_{1}-b_{1} v\right)-3 \tau_{0} \chi_{0}\left(\kappa_{1}\left(\beta_{1}+\theta_{1}\right)+c_{1}+d_{1}\right)\right)-\chi_{0} \tilde{\tau}_{0}\left(a_{1}-b_{1} v\right)\left(\kappa_{2}\left(\beta_{2}+\theta_{2}\right)+c_{2}+d_{2}\right), \\
\mathcal{H}_{2}=3 \chi_{0} \tilde{\tau}_{0}\left(a_{1}-b_{1} v\right)\left(\kappa_{2}\left(\beta_{2}+\theta_{2}\right)+c_{2}+d_{2}\right)+\left(a_{2}-b_{2} v\right)\left(2 \mu_{2} B^{2}\left(a_{1}-b_{1} v\right)+\tau_{0} \chi_{0}\left(\kappa_{1}\left(\beta_{1}+\theta_{1}\right)+c_{1}+d_{1}\right)\right), \\
\mathcal{H}_{3}=\kappa_{1}\left(\beta_{1}+\theta_{1}\right)+c_{1}+d_{1}, \quad \mathcal{H}_{4}=\kappa_{2}\left(\beta_{2}+\theta_{2}\right)+c_{2}+d_{2}, \quad \mathcal{H}_{5}=3 \tau_{1}^{3} \tilde{\tau}_{0}^{3}+3 \tau_{0} \tau_{1}^{2} \tilde{\tau}_{1} \tilde{\tau}_{0}^{2}+3 \tau_{0}^{2} \tau_{1} \tilde{\tau}_{1}^{2} \tilde{\tau}_{0}-\tau_{0}^{3} \tilde{\tau}_{1}^{3}, \\
\mathcal{H}_{6}=-\tau_{1}^{3} \tilde{\tau}_{0}^{3}+3 \tau_{0} \tau_{1}^{2} \tilde{\tau}_{1} \tilde{\tau}_{0}^{2}+3 \tau_{0}^{2} \tau_{1} \tilde{\tau}_{1}^{2} \tilde{\tau}_{0}+3 \tau_{0}^{3} \tilde{\tau}_{1}^{3}, \quad \mathcal{H}_{9}=\tau_{0} \tau_{1} \tilde{\tau}_{1}\left(a_{2}-b_{2} v\right)\left(\mu_{2} B^{2}-4 \kappa_{1}^{2} \chi_{0}\right), \\
\mathcal{H}_{7}=\tau_{1}^{2} \tilde{\tau}_{0}\left(a_{2}-b_{2} v\right)\left(b_{1} \mu_{2} v B^{2}-2 \chi_{0}\left(2 \alpha_{1} \kappa_{1}+3 \tau_{0}\left(\kappa_{1}\left(\beta_{1}+\theta_{1}\right)+c_{1}+d_{1}\right)\right)\right), \\
\mathcal{H}_{8}=\tau_{0} \tau_{1} \tilde{\tau}_{1}\left(b_{2} v-a_{2}\right)\left(b_{1} \mu_{2} v B^{2}-\chi_{0}\left(4 \alpha_{1} \kappa_{1}+3 \tau_{0}\left(\kappa_{1}\left(\beta_{1}+\theta_{1}\right)+c_{1}+d_{1}\right)\right)\right), \\
\mathcal{H}_{10}=\tau_{1} \tilde{\tau}_{0} \tilde{\tau}_{1}\left(a_{1}-b_{1} v\right)\left(\chi_{0}\left(3 \tilde{\tau}_{0}\left(\kappa_{2}\left(\beta_{2}+\theta_{2}\right)+c_{2}+d_{2}\right)+4 \alpha_{2} \kappa_{2}\right)+a_{2}\left(\mu_{2} B^{2}-4 \kappa_{2}^{2} \chi_{0}\right)-b_{2} \mu_{2} v B^{2}\right), \\
\mathcal{H}_{11}=\tau_{0} \tilde{\tau}_{1}^{2}\left(a_{1}-b_{1} v\right)\left(2 \chi_{0}\left(3 \tilde{\tau}_{0}\left(\kappa_{2}\left(\beta_{2}+\theta_{2}\right)+c_{2}+d_{2}\right)+2 \alpha_{2} \kappa_{2}\right)+a_{2}\left(\mu_{2} B^{2}-4 \kappa_{2}^{2} \chi_{0}\right)-b_{2} \mu_{2} v B^{2}\right) .
\end{gathered}
$$

Substituting the solution set (80) into Eqs. (22) and (25), we find that

$$
\pm\left(\xi-\xi_{0}\right)=W \int \frac{d \Psi}{\sqrt{\Lambda(\Psi)}},
$$


where

$$
\Lambda(\Psi)=\Psi^{4}+\frac{\mu_{3}}{\mu_{4}} \Psi^{3}+\frac{\mu_{2}}{\mu_{4}} \Psi^{2}+\frac{\mu_{1}}{\mu_{4}} \Psi+\frac{\mu_{0}}{\mu_{4}}, \quad W=\sqrt{\frac{\chi_{0}}{\mu_{4}}} .
$$

Integrating Eq. (82) and inserting the result into Eqs. (78) and (79), we can write the solution formulas of Eqs. (75) and (76). Then, using the transformation $U_{l}=V_{l}^{\frac{1}{2}}$, we can obtain solutions of Eqs. (71) and (72) as follows:

$$
\begin{aligned}
& U_{1}(\xi)=\left\{\tau_{0}+\tau_{1} \Psi\right\}^{\frac{1}{2}}, \\
& U_{2}(\xi)=\left\{\tilde{\tau}_{0}+\tilde{\tau}_{1} \Psi\right\}^{\frac{1}{2}} .
\end{aligned}
$$

Consequently, we have the following exact solutions to the coupled NLSE in birefringent fibers with GVD and STD (65) and (66):

When $\Lambda(\Psi)=\left(\Psi-\lambda_{1}\right)^{4}$, then we obtain

$$
\begin{gathered}
q(x, t)=\left\{\tau_{0}+\tau_{1} \lambda_{1} \pm \frac{\tau_{1} W}{B\left(x-\frac{2 a_{1} \kappa_{1}-b_{1} \omega_{1}-\alpha_{1}}{b_{1} \kappa_{1}-1} t\right)-\xi_{0}}\right\}^{\frac{1}{2}} \\
\left.\times e^{i\left\{-\kappa_{1} x+\left(\frac{a_{1}\left(\mathcal{H}_{9}-\tau_{1}^{2} \tilde{\tau}_{0}\left(a_{2}-b_{2} v\right)\left(\mu_{2} B^{2}-4 \kappa_{1}^{2} \chi_{0}\right)\right)+3 \tau_{0}^{2} \chi_{0} \tilde{\tau}_{1}^{2} \mathcal{H}_{4}\left(a_{1}-b_{1} v\right)+\mathcal{H}_{7}+\mathcal{H}_{8}}{4 \tau_{1} \chi_{0}\left(\tau_{1} \tilde{\tau}_{0}-\tau_{0} \tilde{\tau}_{1}\right)\left(b_{1} \kappa_{1}-1\right)\left(a_{2}-b_{2} v\right)}\right) t+\sigma_{1}\right.}\right\}, \\
r(x, t)= \\
\left\{\tilde{\tau}_{0}+\tilde{\tau}_{1} \lambda_{1} \pm \frac{\tilde{\tau}_{1} W}{B\left(x-\frac{2 a_{2} \kappa_{2}-b_{2} \omega_{2}-\alpha_{2}}{b_{2} \kappa_{2}-1} t\right)-\xi_{0}}\right\}^{\frac{1}{2}} \\
\times e^{i\left\{-\kappa_{2} x+\left(\frac{3 \tau_{1}^{2} \chi_{0} \tilde{\tau}_{0}^{2} \mathcal{H}_{3}\left(a_{2}-b_{2} v\right)+\mathcal{H}_{10}-\mathcal{H}_{11}}{4 \chi_{0} \tilde{\tau}_{1}\left(\tau_{0} \tilde{\tau}_{1}-\tau_{1} \tilde{\tau}_{0}\right)\left(b_{2} \kappa_{2}-1\right)\left(a_{1}-b_{1} v\right)}\right) t+\sigma_{2}\right\}} .
\end{gathered}
$$

When $\Lambda(\Psi)=\left(\Psi-\lambda_{1}\right)^{3}\left(\Psi-\lambda_{2}\right)$ and $\lambda_{2}>\lambda_{1}$, then we get

$$
\begin{aligned}
& q(x, t)=\left\{\tau_{0}+\tau_{1} \lambda_{1}+\frac{4 W^{2}\left(\lambda_{2}-\lambda_{1}\right) \tau_{1}}{4 W^{2}-\left[\left(\lambda_{1}-\lambda_{2}\right)\left(B\left(x-\frac{2 a_{1} \kappa_{1}-b_{1} \omega_{1}-\alpha_{1}}{b_{1} \kappa_{1}-1} t\right)-\xi_{0}\right)\right]^{2}}\right\}^{\frac{1}{2}} \\
& \times e^{i\left\{-\kappa_{1} x+\left(\frac{a_{1}\left(\mathcal{H}_{9}-\tau_{1}^{2} \tilde{\tau}_{0}\left(a_{2}-b_{2} v\right)\left(\mu_{2} B^{2}-4 \kappa_{1}^{2} \chi_{0}\right)\right)+3 \tau_{0}^{2} \chi_{0} \tilde{\tau}_{1}^{2} \mathcal{H}_{4}\left(a_{1}-b_{1} v\right)+\mathcal{H}_{7}+\mathcal{H}_{8}}{4 \tau_{1} \chi_{0}\left(\tau_{1} \tilde{\tau}_{0}-\tau_{0} \tilde{\tau}_{1}\right)\left(b_{1} \kappa_{1}-1\right)\left(a_{2}-b_{2} v\right)}\right) t+\sigma_{1}\right\}}, \\
& r(x, t)=\left\{\tilde{\tau}_{0}+\tilde{\tau}_{1} \lambda_{1}+\frac{4 W^{2}\left(\lambda_{2}-\lambda_{1}\right) \tilde{\tau}_{1}}{4 W^{2}-\left[\left(\lambda_{1}-\lambda_{2}\right)\left(B\left(x-\frac{2 a_{2} \kappa_{2}-b_{2} \omega_{2}-\alpha_{2}}{b_{2} \kappa_{2}-1} t\right)-\xi_{0}\right)\right]^{2}}\right\}^{\frac{1}{2}} \\
& \times e^{i\left\{-\kappa_{2} x+\left(\frac{3 \tau_{1}^{2} \chi_{0} \tilde{\tau}_{0}^{2} \mathcal{H}_{3}\left(a_{2}-b_{2} v\right)+\mathcal{H}_{10}-\mathcal{H}_{11}}{4 \chi_{0} \tilde{\tau}_{1}\left(\tau_{0} \tilde{\tau}_{1}-\tau_{1} \tilde{\tau}_{0}\right)\left(b_{2} \kappa_{2}-1\right)\left(a_{1}-b_{1} v\right)}\right) t+\sigma_{2}\right\}} .
\end{aligned}
$$

When $\Lambda(\Psi)=\left(\Psi-\lambda_{1}\right)^{2}\left(\Psi-\lambda_{2}\right)^{2}$, then we have

$$
\begin{aligned}
q(x, t)= & \left\{\tau_{0}+\tau_{1} \lambda_{2}+\frac{\left(\lambda_{2}-\lambda_{1}\right) \tau_{1}}{\exp \left[\frac{\lambda_{1}-\lambda_{2}}{W}\left(B\left(x-\frac{2 a_{1} \kappa_{1}-b_{1} \omega_{1}-\alpha_{1}}{b_{1} \kappa_{1}-1} t\right)-\xi_{0}\right)\right]-1}\right\}^{\frac{1}{2}} \\
& \times e^{i\left\{-\kappa_{1} x+\left(\frac{a_{1}\left(\mathcal{H}_{9}-\tau_{1}^{2} \tilde{\tau}_{0}\left(a_{2}-b_{2} v\right)\left(\mu_{2} B^{2}-4 \kappa_{1}^{2} \chi_{0}\right)\right)+3 \tau_{0}^{2} \chi_{0} \tilde{\tau}_{1}^{2} \mathcal{H}_{4}\left(a_{1}-b_{1} v\right)+\mathcal{H}_{7}+\mathcal{H}_{8}}{4 \tau_{1} \chi_{0}\left(\tau_{1} \tilde{\tau}_{0}-\tau_{0} \tilde{\tau}_{1}\right)\left(b_{1} \kappa_{1}-1\right)\left(a_{2}-b_{2} v\right)}\right) t+\sigma_{1}\right\}},
\end{aligned}
$$




$$
\begin{aligned}
r(x, t)= & \left\{\tilde{\tau}_{0}+\tilde{\tau}_{1} \lambda_{2}+\frac{\left(\lambda_{2}-\lambda_{1}\right) \tilde{\tau}_{1}}{\exp \left[\frac{\lambda_{1}-\lambda_{2}}{W}\left(B\left(x-\frac{2 a_{2} \kappa_{2}-b_{2} \omega_{2}-\alpha_{2}}{b_{2} \kappa_{2}-1} t\right)-\xi_{0}\right)\right]-1}\right\}^{\frac{1}{2}} \\
& \times e^{i\left\{-\kappa_{2} x+\left(\frac{3 \tau_{1}^{2} \chi_{0} \tilde{\tau}_{0}^{2} \mathcal{H}_{3}\left(a_{2}-b_{2} v\right)+\mathcal{H}_{10}-\mathcal{H}_{11}}{4 \chi_{0} \tilde{\tau}_{1}\left(\tau_{0} \tilde{\tau}_{1}-\tau_{1} \tilde{\tau}_{0}\right)\left(b_{2} \kappa_{2}-1\right)\left(a_{1}-b_{1} v\right)}\right) t+\sigma_{2}\right\}},
\end{aligned}
$$

and

$$
\begin{aligned}
& q(x, t)=\left\{\tau_{0}+\tau_{1} \lambda_{1}+\frac{\left(\lambda_{1}-\lambda_{2}\right) \tau_{1}}{\exp \left[\frac{\lambda_{1}-\lambda_{2}}{W}\left(B\left(x-\frac{2 a_{1} \kappa_{1}-b_{1} \omega_{1}-\alpha_{1}}{b_{1} \kappa_{1}-1} t\right)-\xi_{0}\right)\right]-1}\right\}^{\frac{1}{2}} \\
& \times e^{i\left\{-\kappa_{1} x+\left(\frac{a_{1}\left(\mathcal{H}_{9}-\tau_{1}^{2} \tilde{\tau}_{0}\left(a_{2}-b_{2} v\right)\left(\mu_{2} B^{2}-4 \kappa_{1}^{2} \chi_{0}\right)\right)+3 \tau_{0}^{2} \chi_{0} \tilde{\tau}_{1}^{2} \mathcal{H}_{4}\left(a_{1}-b_{1} v\right)+\mathcal{H}_{7}+\mathcal{H}_{8}}{4 \tau_{1} \chi_{0}\left(\tau_{1} \tilde{\tau}_{0}-\tau_{0} \tilde{\tau}_{1}\right)\left(b_{1} \kappa_{1}-1\right)\left(a_{2}-b_{2} v\right)}\right) t+\sigma_{1}\right\}}, \\
& r(x, t)=\left\{\tilde{\tau}_{0}+\tilde{\tau}_{1} \lambda_{1}+\frac{\left(\lambda_{1}-\lambda_{2}\right) \tilde{\tau}_{1}}{\exp \left[\frac{\lambda_{1}-\lambda_{2}}{W}\left(B\left(x-\frac{2 a_{2} \kappa_{2}-b_{2} \omega_{2}-\alpha_{2}}{b_{2} \kappa_{2}-1} t\right)-\xi_{0}\right)\right]-1}\right\}^{\frac{1}{2}} \\
& \times e^{i\left\{-\kappa_{2} x+\left(\frac{3 \tau_{1}^{2} \chi_{0} \tilde{\tau}_{0}^{2} \mathcal{H}_{3}\left(a_{2}-b_{2} v\right)+\mathcal{H}_{10}-\mathcal{H}_{11}}{4 \chi_{0} \tilde{\tau}_{1}\left(\tau_{0} \tilde{\tau}_{1}-\tau_{1} \tilde{\tau}_{0}\right)\left(b_{2} \kappa_{2}-1\right)\left(a_{1}-b_{1} v\right)}\right) t+\sigma_{2}\right\}} .
\end{aligned}
$$

When $\Lambda(\Psi)=\left(\Psi-\lambda_{1}\right)^{2}\left(\Psi-\lambda_{2}\right)\left(\Psi-\lambda_{3}\right)$ and $\lambda_{1}>\lambda_{2}>\lambda_{3}$, then we attain

$$
\begin{aligned}
q(x, t)= & \left\{\tau_{0}+\tau_{1} \lambda_{1}-\frac{2\left(\lambda_{1}-\lambda_{2}\right)\left(\lambda_{1}-\lambda_{3}\right) \tau_{1}}{2 \lambda_{1}-\lambda_{2}-\lambda_{3}+\left(\lambda_{3}-\lambda_{2}\right) \cosh \left(\frac{\sqrt{\left(\lambda_{1}-\lambda_{2}\right)\left(\lambda_{1}-\lambda_{3}\right)}}{W}\left[B\left(x-\frac{2 a_{1} \kappa_{1}-b_{1} \omega_{1}-\alpha_{1}}{b_{1} \kappa_{1}-1} t\right)\right]\right)}\right\}^{\frac{1}{2}} \\
& \times e^{i\left\{-\kappa_{1} x+\left(\frac{a_{1}\left(\mathcal{H}_{9}-\tau_{1}^{2} \tilde{\tau}_{0}\left(a_{2}-b_{2} v\right)\left(\mu_{2} B^{2}-\kappa_{1}^{2} \chi_{0}\right)\right)+3 \tau_{0}^{2} \chi_{0} \tilde{\tau}_{1}^{2} \mathcal{H}_{4}\left(a_{1}-b_{1} v\right)+\mathcal{H}_{7}+\mathcal{H}_{8}}{4 \tau_{1} \chi_{0}\left(\tau_{1} \tilde{\tau}_{0}-\tau_{0} \tilde{\tau}_{1}\right)\left(b_{1} \kappa_{1}-1\right)\left(a_{2}-b_{2} v\right)}\right) t+\sigma_{1}\right\}}, \\
r(x, t)= & \left\{\begin{array}{l}
\tilde{\tau}_{0}+\tilde{\tau}_{1} \lambda_{1}-\frac{2\left(\lambda_{1}-\lambda_{2}\right)\left(\lambda_{1}-\lambda_{3}\right)}{2 \lambda_{1}-\tilde{\tau}_{1}} \\
\end{array}\right. \\
& \times e^{i\left\{-\kappa_{2} x+\left(\frac{3 \tau_{1}^{2} \chi_{0} \tilde{\tau}_{0}^{2} \mathcal{H}_{3}\left(a_{2}-b_{2} v\right)+\mathcal{H}_{10}-\mathcal{H}_{11}}{4 \chi_{0} \tilde{\tau}_{1}\left(\tau_{0} \tilde{\tau}_{1}-\tau_{1} \tilde{\tau}_{0}\right)\left(b_{2} \kappa_{2}-1\right)\left(a_{1}-b_{1} v\right)}\right) t+\sigma_{2}\right\}} .
\end{aligned}
$$

When $\Lambda(\Psi)=\left(\Psi-\lambda_{1}\right)\left(\Psi-\lambda_{2}\right)\left(\Psi-\lambda_{3}\right)\left(\Psi-\lambda_{4}\right)$ and $\lambda_{1}>\lambda_{2}>\lambda_{3}>\lambda_{4}$, then we achieve

$$
\begin{aligned}
& q(x, t)=\left\{\tau_{0}+\tau_{1} \lambda_{2}+\frac{\tau_{1}\left(\lambda_{1}-\lambda_{2}\right)\left(\lambda_{4}-\lambda_{2}\right)}{\lambda_{4}-\lambda_{2}+\left(\lambda_{1}-\lambda_{4}\right) \operatorname{sn}^{2}\left[ \pm \frac{\sqrt{\left(\lambda_{1}-\lambda_{3}\right)\left(\lambda_{2}-\lambda_{4}\right)}}{2 W}\left[B\left(x-\frac{2 a_{1} \kappa_{1}-b_{1} \omega_{1}-\alpha_{1}}{b_{1} \kappa_{1}-1} t\right)-\xi_{0}\right], l\right]}\right\}^{\frac{1}{2}} \\
& \times e^{i\left\{-\kappa_{1} x+\left(\frac{a_{1}\left(\mathcal{H}_{9}-\tau_{1}^{2} \tilde{\tau}_{0}\left(a_{2}-b_{2} v\right)\left(\mu_{2} B^{2}-4 \kappa_{1}^{2} \chi_{0}\right)\right)+3 \tau_{0}^{2} \chi_{0} \tilde{\tau}_{1}^{2} \mathcal{H}_{4}\left(a_{1}-b_{1} v\right)+\mathcal{H}_{7}+\mathcal{H}_{8}}{4 \tau_{1} \chi_{0}\left(\tau_{1} \tilde{\tau}_{0}-\tau_{0} \tilde{\tau}_{1}\right)\left(b_{1} \kappa_{1}-1\right)\left(a_{2}-b_{2} v\right)}\right) t+\sigma_{1}\right\}},
\end{aligned}
$$




$$
\begin{aligned}
r(x, t)= & \left\{\begin{array}{c}
\tilde{\tau}_{0}+\tilde{\tau}_{1} \lambda_{2}+\frac{\tilde{\tau}_{1}\left(\lambda_{1}-\lambda_{2}\right)\left(\lambda_{4}-\lambda_{2}\right)}{\lambda_{4}-\lambda_{2}+\left(\lambda_{1}-\lambda_{4}\right) \mathrm{sn}^{2}\left[ \pm \frac{\sqrt{\left(\lambda_{1}-\lambda_{3}\right)\left(\lambda_{2}-\lambda_{4}\right)}}{2 W}\left[B\left(x-\frac{2 a_{2} \kappa_{2}-b_{2} \omega_{2}-\alpha_{2}}{b_{2} \kappa_{2}-1} t\right)-\xi_{0}\right], l\right]} \\
\underbrace{i\left\{-\kappa_{2} x+\left(\frac{3 \tau_{1}^{2} \chi_{0} \tilde{\tau}_{0}^{2} \mathcal{H}_{3}\left(a_{2}-b_{2} v\right)+\mathcal{H}_{10}-\mathcal{H}_{11}}{4 \chi_{0} \tilde{\tau}_{1}\left(\tau_{0} \tilde{\tau}_{1}-\tau_{1} \tilde{\tau}_{0}\right)\left(b_{2} \kappa_{2}-1\right)\left(a_{1}-b_{1} v\right)}\right) t+\sigma_{2}\right\}}
\end{array}\right. \\
& \times e^{\frac{1}{2}}
\end{aligned}
$$

where

$$
l^{2}=\frac{\left(\lambda_{2}-\lambda_{3}\right)\left(\lambda_{1}-\lambda_{4}\right)}{\left(\lambda_{1}-\lambda_{3}\right)\left(\lambda_{2}-\lambda_{4}\right)} .
$$

Also, $\lambda_{i}(i=1, \ldots, 4)$ are the roots of the polynomial equation

$$
\Lambda(\Psi)=0 .
$$

When $\tau_{0}=-\tau_{1} \lambda_{1}, \tilde{\tau}_{0}=-\tilde{\tau}_{1} \lambda_{1}$ and $\xi_{0}=0$, then we can reduce the solutions (86)-(95) to plane wave solutions

$$
\begin{aligned}
& \left.q(x, t)=\left\{ \pm \frac{\tau_{1} W}{B\left(x-\frac{2 a_{1} \kappa_{1}-b_{1} \omega_{1}-\alpha_{1}}{b_{1} \kappa_{1}-1} t\right)}\right\}^{\frac{1}{2}} e^{i\left\{-\kappa_{1} x+\left(\frac{a_{1}\left(\mathcal{H}_{9}-\tau_{1}^{2} \tilde{\tau}_{0}\left(a_{2}-b_{2} v\right)\left(\mu_{2} B^{2}-4 \kappa_{1}^{2} \chi_{0}\right)\right)+3 \tau_{0}^{2} \chi_{0} \tilde{\tau}_{1}^{2} \mathcal{H}_{4}\left(a_{1}-b_{1} v\right)+\mathcal{H}_{7}+\mathcal{H}_{8}}{4 \tau_{1} \chi_{0}\left(\tau_{1} \tilde{\tau}_{0}-\tau_{0} \tilde{\tau}_{1}\right)\left(b_{1} \kappa_{1}-1\right)\left(a_{2}-b_{2} v\right)}\right) t+\sigma_{1}\right.}\right\}, \\
& r(x, t)=\left\{ \pm \frac{\tilde{\tau}_{1} W}{B\left(x-\frac{2 a_{2} \kappa_{2}-b_{2} \omega_{2}-\alpha_{2}}{b_{2} \kappa_{2}-1} t\right)}\right\}^{\frac{1}{2}} e^{i\left\{-\kappa_{2} x+\left(\frac{3 \tau_{1}^{2} \chi_{0} \tilde{\tau}_{0}^{2} \mathcal{H}_{3}\left(a_{2}-b_{2} v\right)+\mathcal{H}_{10}-\mathcal{H}_{11}}{4 \chi_{0} \tilde{\tau}_{1}\left(\tau_{0} \tilde{\tau}_{1}-\tau_{1} \tilde{\tau}_{0}\right)\left(b_{2} \kappa_{2}-1\right)\left(a_{1}-b_{1} v\right)}\right) t+\sigma_{2}\right\}}, \\
& q(x, t)=\left\{\frac{4 W^{2}\left(\lambda_{2}-\lambda_{1}\right) \tau_{1}}{4 W^{2}-\left[\left(\lambda_{1}-\lambda_{2}\right)\left(B\left(x-\frac{2 a_{1} \kappa_{1}-b_{1} \omega_{1}-\alpha_{1}}{b_{1} \kappa_{1}-1} t\right)\right)\right]^{2}}\right\}^{\frac{1}{2}} \\
& \times e^{i\left\{-\kappa_{1} x+\left(\frac{a_{1}\left(\mathcal{H}_{9}-\tau_{1}^{2} \tilde{\tau}_{0}\left(a_{2}-b_{2} v\right)\left(\mu_{2} B^{2}-4 \kappa_{1}^{2} \chi_{0}\right)\right)+3 \tau_{0}^{2} \chi_{0} \tilde{\tau}_{1}^{2} \mathcal{H}_{4}\left(a_{1}-b_{1} v\right)+\mathcal{H}_{7}+\mathcal{H}_{8}}{4 \tau_{1} \chi_{0}\left(\tau_{1} \tilde{\tau}_{0}-\tau_{0} \tilde{\tau}_{1}\right)\left(b_{1} \kappa_{1}-1\right)\left(a_{2}-b_{2} v\right)}\right) t+\sigma_{1}\right\}}, \\
& r(x, t)=\left\{\frac{4 W^{2}\left(\lambda_{2}-\lambda_{1}\right) \tilde{\tau}_{1}}{4 W^{2}-\left[\left(\lambda_{1}-\lambda_{2}\right)\left(B\left(x-\frac{2 a_{2} \kappa_{2}-b_{2} \omega_{2}-\alpha_{2}}{b_{2} \kappa_{2}-1} t\right)\right)\right]^{2}}\right\}^{\frac{1}{2}} \\
& \times e^{i\left\{-\kappa_{2} x+\left(\frac{3 \tau_{1}^{2} \chi_{0} \tilde{\tau}_{0}^{2} \mathcal{H}_{3}\left(a_{2}-b_{2} v\right)+\mathcal{H}_{10}-\mathcal{H}_{11}}{4 \chi_{0} \tilde{\tau}_{1}\left(\tau_{0} \tilde{\tau}_{1}-\tau_{1} \tilde{\tau}_{0}\right)\left(b_{2} \kappa_{2}-1\right)\left(a_{1}-b_{1} v\right)}\right) t+\sigma_{2}\right\}},
\end{aligned}
$$

singular soliton solutions

$$
\begin{aligned}
q(x, t)= & \frac{\left(\lambda_{2}-\lambda_{1}\right) \tau_{1}}{2}\left\{1 \mp \operatorname{coth}\left[\frac{\lambda_{1}-\lambda_{2}}{2 W}\left(B\left(x-\frac{2 a_{1} \kappa_{1}-b_{1} \omega_{1}-\alpha_{1}}{b_{1} \kappa_{1}-1} t\right)\right)\right]\right\}^{\frac{1}{2}} \\
& \times e^{i\left\{-\kappa_{1} x+\left(\frac{a_{1}\left(\mathcal{H}_{9}-\tau_{1}^{2} \tilde{\tau}_{0}\left(a_{2}-b_{2} v\right)\left(\mu_{2} B^{2}-4 \kappa_{1}^{2} \chi_{0}\right)\right)+3 \tau_{0}^{2} \chi_{0} \tilde{\tau}_{1}^{2} \mathcal{H}_{4}\left(a_{1}-b_{1} v\right)+\mathcal{H}_{7}+\mathcal{H}_{8}}{4 \tau_{1} \chi_{0}\left(\tau_{1} \tilde{\tau}_{0}-\tau_{0} \tilde{\tau}_{1}\right)\left(b_{1} \kappa_{1}-1\right)\left(a_{2}-b_{2} v\right)}\right) t+\sigma_{1}\right\}}, \\
r(x, t)= & \frac{\left(\lambda_{2}-\lambda_{1}\right) \tilde{\tau}_{1}}{2}\left\{1 \mp \operatorname{coth}\left[\frac{\lambda_{1}-\lambda_{2}}{2 W}\left(B\left(x-\frac{2 a_{2} \kappa_{2}-b_{2} \omega_{2}-\alpha_{2}}{b_{2} \kappa_{2}-1} t\right)\right)\right]\right\}^{\frac{1}{2}} \\
& \times e^{i\left\{-\kappa_{2} x+\left(\frac{3 \tau_{1}^{2} \chi_{0} \tilde{\tau}_{0}^{2} \mathcal{H}_{3}\left(a_{2}-b_{2} v\right)+\mathcal{H}_{10}-\mathcal{H}_{11}}{4 \chi_{0} \tilde{\tau}_{1}\left(\tau_{0} \tilde{\tau}_{1}-\tau_{1} \tilde{\tau}_{0}\right)\left(b_{2} \kappa_{2}-1\right)\left(a_{1}-b_{1} v\right)}\right) t+\sigma_{2}\right\}},
\end{aligned}
$$


and bright soliton solutions

$$
\begin{gathered}
q(x, t)=\left\{\frac{A}{C+\cosh \left[D\left(B\left(x-\frac{2 a_{1} \kappa_{1}-b_{1} \omega_{1}-\alpha_{1}}{b_{1} \kappa_{1}-1} t\right)\right)\right]}\right\}^{\frac{1}{2}} \\
\left.\times e^{i\left\{-\kappa_{1} x+\left(\frac{a_{1}\left(\mathcal{H}_{9}-\tau_{1}^{2} \tilde{\tau}_{0}\left(a_{2}-b_{2} v\right)\left(\mu_{2} B^{2}-4 \kappa_{1}^{2} \chi_{0}\right)\right)+3 \tau_{0}^{2} \chi_{0} \tilde{\tau}_{1}^{2} \mathcal{H}_{4}\left(a_{1}-b_{1} v\right)+\mathcal{H}_{7}+\mathcal{H}_{8}}{\tau_{1} \chi_{0}\left(\tau_{1} \tilde{\tau}_{0}-\tau_{0} \tilde{\tau}_{1}\right)\left(b_{1} \kappa_{1}-1\right)\left(a_{2}-b_{2} v\right)}\right) t+\sigma_{1}\right.}\right\}, \\
r(x, t)=\left\{\frac{\tilde{A}}{C+\cosh \left[D\left(B\left(x-\frac{2 a_{2} \kappa_{2}-b_{2} \omega_{2}-\alpha_{2}}{b_{2} \kappa_{2}-1} t\right)\right)\right]}\right\}^{\frac{1}{2}} \\
\times e^{i\left\{-\kappa_{2} x+\left(\frac{3 \tau_{1}^{2} \chi_{0} \tilde{\tau}_{2}^{2} \mathcal{H}_{3}\left(a_{2}-b_{2} v\right)+\mathcal{H}_{10}-\mathcal{H}_{11}}{4 \chi_{0} \tilde{\tau}_{1}\left(\tau_{0} \tilde{\tau}_{1}-\tau_{1} \tilde{\tau}_{0}\right)\left(b_{2} \kappa_{2}-1\right)\left(a_{1}-b_{1} v\right)}\right) t+\sigma_{2}\right\}},
\end{gathered}
$$

where

$$
A=\frac{2\left(\lambda_{1}-\lambda_{2}\right)\left(\lambda_{1}-\lambda_{3}\right) \tau_{1}}{\lambda_{3}-\lambda_{2}}, \quad \tilde{A}=\frac{2\left(\lambda_{1}-\lambda_{2}\right)\left(\lambda_{1}-\lambda_{3}\right) \tilde{\tau}_{1}}{\lambda_{3}-\lambda_{2}}, \quad D=\frac{\sqrt{\left(\lambda_{1}-\lambda_{2}\right)\left(\lambda_{1}-\lambda_{3}\right)}}{W}, \quad C=\frac{2 \lambda_{1}-\lambda_{2}-\lambda_{3}}{\lambda_{3}-\lambda_{2}} .
$$

Here, $A$ and $\tilde{A}$ are the amplitudes of the solitons, while $D$ is related to inverse width of the solitons. These solitons exist for $\tau_{1}<0$ and $\tilde{\tau}_{1}<0$. Moreover, when $\tau_{0}=-\tau_{1} \lambda_{2}, \tilde{\tau}_{0}=-\tilde{\tau}_{1} \lambda_{2}$ and $\xi_{0}=0$, we can write the Jacobi elliptic function solutions (96) and (97) as follows:

$$
\begin{aligned}
q(x, t)= & \left\{\frac{A_{1}}{C_{1}+\mathrm{sn}^{2}\left[D_{j}\left[B\left(x-\frac{2 a_{1} \kappa_{1}-b_{1} \omega_{1}-\alpha_{1}}{b_{1} \kappa_{1}-1} t\right)\right], \frac{\left(\lambda_{2}-\lambda_{3}\right)\left(\lambda_{1}-\lambda_{4}\right)}{\left(\lambda_{1}-\lambda_{3}\right)\left(\lambda_{2}-\lambda_{4}\right)}\right\}^{\frac{1}{2}}}\right\} \\
\times & \left.\times e^{i\left\{-\kappa_{1} x+\left(\frac{a_{1}\left(\mathcal{H}_{9}-\tau_{1}^{2} \tilde{\tau}_{0}\left(a_{2}-b_{2} v\right)\left(\mu_{2} B^{2}-4_{1}^{2} \chi_{0}\right)\right)+3 \tau_{0}^{2} \chi_{0} \tilde{\tau}_{1}^{2} \mathcal{H}_{4}\left(a_{1}-b_{1} v\right)+\mathcal{H}_{7}+\mathcal{H}_{8}}{4 \tau_{1} \chi_{0}\left(\tau_{1} \tilde{\tau}_{0}-\tau_{0} \tilde{\tau}_{1}\right)\left(b_{1} \kappa_{1}-1\right)\left(a_{2}-b_{2} v\right)}\right) t+\sigma_{1}\right.}\right\}, \\
r(x, t)= & \left\{\frac{\tilde{A}_{1}}{C_{1}+\operatorname{sn}^{2}\left[D_{j}\left[B\left(x-\frac{2 a_{2} \kappa_{2}-b_{2} \omega_{2}-\alpha_{2}}{b_{2} \kappa_{2}-1} t\right)\right], \frac{\left(\lambda_{2}-\lambda_{3}\right)\left(\lambda_{1}-\lambda_{4}\right)}{\left(\lambda_{1}-\lambda_{3}\right)\left(\lambda_{2}-\lambda_{4}\right)}\right]}\right\}^{\frac{1}{2}} \\
& \times e^{i\left\{-\kappa_{2} x+\left(\frac{3 \tau_{1}^{2} \chi_{0} \tilde{\tau}_{0}^{2} \mathcal{H}_{3}\left(a_{2}-b_{2} v\right)+\mathcal{H}_{10}-\mathcal{H}_{11}}{4 \chi_{0} \tilde{\tau}_{1}\left(\tau_{0} \tilde{\tau}_{1}-\tau_{1} \tilde{\tau}_{0}\right)\left(b_{2} \kappa_{2}-1\right)\left(a_{1}-b_{1} v\right)}\right) t+\sigma_{2}\right\}},
\end{aligned}
$$

where

$$
\begin{gathered}
A_{1}=\frac{\tau_{1}\left(\lambda_{1}-\lambda_{2}\right)\left(\lambda_{4}-\lambda_{2}\right)}{\lambda_{1}-\lambda_{4}}, \quad \tilde{A}_{1}=\frac{\tilde{\tau}_{1}\left(\lambda_{1}-\lambda_{2}\right)\left(\lambda_{4}-\lambda_{2}\right)}{\lambda_{1}-\lambda_{4}}, \\
C_{1}=\frac{\lambda_{4}-\lambda_{2}}{\lambda_{1}-\lambda_{4}}, \quad D_{j}=\frac{(-1)^{j} \sqrt{\left(\lambda_{1}-\lambda_{3}\right)\left(\lambda_{2}-\lambda_{4}\right)}}{2 W}, \quad(j=1,2) .
\end{gathered}
$$

$\underline{\text { Remark-1: }}$ When the modulus $l \rightarrow 1$, a second form of singular optical soliton solutions fall out:

$$
\begin{aligned}
q(x, t)= & \left\{\frac{A_{1}}{C_{1}+\tanh ^{2}\left[D_{j}\left(B\left(x-\frac{2 a_{1} \kappa_{1}-b_{1} \omega_{1}-\alpha_{1}}{b_{1} \kappa_{1}-1} t\right)\right)\right]}\right\}^{\frac{1}{2}} \\
& \left.\times e^{i\left\{-\kappa_{1} x+\left(\frac{a_{1}\left(\mathcal{H}_{9}-\tau_{1}^{2} \tilde{\tau}_{0}\left(a_{2}-b_{2} v\right)\left(\mu_{2} B^{2}-4 \kappa_{1}^{2} \chi_{0}\right)\right)+3 \tau_{0}^{2} \chi_{0} \tilde{\tau}_{1}^{2} \mathcal{H}_{4}\left(a_{1}-b_{1} v\right)+\mathcal{H}_{7}+\mathcal{H}_{8}}{4 \tau_{1} \chi_{0}\left(\tau_{1} \tilde{\tau}_{0}-\tau_{0} \tilde{\tau}_{1}\right)\left(b_{1} \kappa_{1}-1\right)\left(a_{2}-b_{2} v\right)}\right) t+\sigma_{1}\right.}\right\},
\end{aligned}
$$




$$
\begin{aligned}
r(x, t)= & \left\{\frac{\tilde{A}_{1}}{C_{1}+\tanh ^{2}\left[D_{j}\left(B\left(x-\frac{2 a_{2} \kappa_{2}-b_{2} \omega_{2}-\alpha_{2}}{b_{2} \kappa_{2}-1} t\right)\right)\right]}\right\}^{\frac{1}{2}} \\
& \times e^{i\left\{-\kappa_{2} x+\left(\frac{3 \tau_{1}^{2} \chi_{0} \tilde{\tau}_{0}^{2} \mathcal{H}_{3}\left(a_{2}-b_{2} v\right)+\mathcal{H}_{10}-\mathcal{H}_{11}}{4 \chi_{0} \tilde{\tau}_{1}\left(\tau_{0} \tilde{\tau}_{1}-\tau_{1} \tilde{\tau}_{0}\right)\left(b_{2} \kappa_{2}-1\right)\left(a_{1}-b_{1} v\right)}\right) t+\sigma_{2}\right\}},
\end{aligned}
$$

where $\lambda_{3}=\lambda_{4}$.

$\underline{\text { Remark-2: }}$ However, if $l \rightarrow 0$, periodic singular solutions are as listed below:

$$
\begin{gathered}
q(x, t)=\left\{\frac{A_{1}}{C_{1}+\sin ^{2}\left[D_{j}\left(B\left(x-\frac{2 a_{1} \kappa_{1}-b_{1} \omega_{1}-\alpha_{1}}{b_{1} \kappa_{1}-1} t\right)\right)\right]}\right\}^{\frac{1}{2}} \\
\times e^{i\left\{-\kappa_{1} x+\left(\frac{a_{1}\left(\mathcal{H}_{9}-\tau_{1}^{2} \tilde{\tau}_{0}\left(a_{2}-b_{2} v\right)\left(\mu_{2} B^{2}-4 \kappa_{1}^{2} \chi_{0}\right)\right)+3 \tau_{0}^{2} \chi_{0} \tilde{\tau}_{1}^{2} \mathcal{H}_{4}\left(a_{1}-b_{1} v\right)+\mathcal{H}_{7}+\mathcal{H}_{8}}{4 \tau_{1} \chi_{0}\left(\tau_{1} \tilde{\tau}_{0}-\tau_{0} \tilde{\tau}_{1}\right)\left(b_{1} \kappa_{1}-1\right)\left(a_{2}-b_{2} v\right)}\right) t+\sigma_{1}\right\}}, \\
r(x, t)=\left\{\frac{\tilde{A}_{1}}{C_{1}+\sin ^{2}\left[D_{j}\left(B\left(x-\frac{2 a_{2} \kappa_{2}-b_{2} \omega_{2}-\alpha_{2}}{b_{2} \kappa_{2}-1}\right)\right)\right]}\right\}^{\frac{1}{2}} \\
\times e^{i\left\{-\kappa_{2} x+\left(\frac{3 \tau_{1}^{2} \chi_{0} \tilde{\tau}_{0}^{2} \mathcal{H}_{3}\left(a_{2}-b_{2} v\right)+\mathcal{H}_{10}-\mathcal{H}_{11}}{4 \chi_{0} \tilde{\tau}_{1}\left(\tau_{0} \tau_{1}-\tau_{1} \tilde{\tau}_{0}\right)\left(b_{2} \kappa_{2}-1\right)\left(a_{1}-b_{1} v\right)}\right) t+\sigma_{2}\right\}},
\end{gathered}
$$

where $\lambda_{2}=\lambda_{3}$.

\section{CONCLUSIONS}

This paper carried out a comprehensive study of optical solitons in birefringent fibers by the aid of extended trial function method that is indeed a powerful integration tool. This algorithm led to bright and singular optical soliton solutions for birefringent fibers with Kerr and parabolic law nonlinearity. There are constraint conditions that naturally emerged from the algebraic structure of solutions and these conditions are indeed mandated for the soliton solutions to exist. There are several other solutions that fell out from the parameter connections. These are singular periodic solutions and doubly periodic solutions.

This paper stands on a strong footing for additional investigation in this subject matter. The FWM terms are to be included. Later, the study will be extended to DWDM systems with FWM and other perturbation terms. Other integration tools will be applied to obtain additional solutions. These are Lie symmetry analysis, mapping method, Kudryashov's method and several others. Those results will be disseminated soon.

\section{ACKNOWLEDGMENTS}

The fourth author (QZ) was funded by the National Science Foundation of Hubei Province in China under the grant number 2015CFC891. The sixth author (SPM) would like to thank the research support provided by the Department of Mathematics and Statistics at Tshwane University of Technology and the support from the South African National Foundation under Grant Number 92052 IRF1202210126. The seventh author (AB) would like to thank Tshwane University of Technology during his academic visit on 2016. The research work of seventh and eighth authors (AB \& MB) were supported by Qatar National Research Fund (QNRF) under the grant number NPRP 6-021-1-005. The authors also declare that there is no conflict of interest. 


\section{References}

[1] A. H. Bhrawy, A. A. Alshaery, E. M. Hilal, M. Savescu, D. Milovic, K. R. Khan, M. F. Mahmood, Z. Jovanoski $\&$ A. Biswas. "Optical solitons in birefringent fibers with spatio-temporal dispersion". Optik. Volume 125, Issue 17, 4935-4944. (2014).

[2] A. Biswas, K. Khan, A. Rahman, A. Yildirim, T. Hayat \& O. M. Aldossary. "Bright and dark optical solitons in birefringent fibers with Hamiltonian perturbations and Kerr law nonlinearity". Journal of Optoelectronics and Advanced Materials. Volume 14, Issues 7-8, 571-576. (2012).

[3] Y. H. Chen, J. L. Wang \& W. X. Tian. "Soliton coupling in birefringent optical fiber with third order dispersion". Optik. Volume 120, Issue 5, 212-215. (2009).

[4] R. K. Dowluru \& P. K. Bhima. "Influence of third order dispersion on linear birefringent optical soliton transmission system". Journal of Optics. Volume 40, Issue 3, 132-142. (2011).

[5] M. Ekici, M. Mirzazadeh \& M. Eslami. "Solitons and other solutions to Boussinesq equation with power law nonlinearity and dual dispersion". Nonlinear Dynamics. doi:10.1007/s11071-015-2515-1

[6] A. Filiz, A. Sonmezoglu, M. Ekici \& D. Duran. "A new approach for soliton solutions of RLW equation and (1+2)-dimensional nonlinear Schrödinger's equation". Mathematical Reports. Volume 17, No. 67, 43-56. (2015).

[7] A. S. Fokas. "On a class of physically important integral equations". Physica D. Volume 87, Issues 1-4, 145-150. (1995).

[8] X. Geng \& Y. Lv. "Darboux transformation for an integrable generalization of the nonlinear Schrödinger equation". Nonlinear Dynamics. Volume 69, Issue 4, 1621-1630. (2012).

[9] S. Kumar, K. Singh \& R. K. Gupta. "Coupled Higgs field equation and Hamiltonian amplitude equation: Lie classical approach and $G^{\prime} / G$ - expansion method". Pramana. Volume 79, Issue 1, 41-60. (2012).

[10] C. S. Liu. "Travelling Wave Solutions of Triple Sine-Gordon Equation". Chinese Physics Letters. Volume 21, No. 12, 2369. (2004).

[11] C. S. Liu. "All Single Traveling Wave Solutions to (3+ 1)-Dimensional Nizhnik-Novikov-Veselov Equation". Communications in Theoretical Physics. Volume 45, 991-992. (2006).

[12] C. S. Liu. "Exact traveling wave solutions for a kind of generalized Ginzburg-Landau equation". Communications in Theoretical Physics. Volume 43, No. 5, 787. (2005).

[13] C. S. Liu. "Classification of all single travelling wave solutions to Calogero-Degasperis-Focas equation". Communications in Theoretical Physics. Volume 48, No. 4, 601. (2007).

[14] C. S. Liu. "Representations and classification of traveling wave solutions to sinh-Gordon equation". Communications in Theoretical Physics. Volume 49, No. 1, 153. (2008).

[15] C. S. Liu. "Applications of complete discrimination system for polynomial for classifications of traveling wave solutions to nonlinear differential equations". Computer Physics Communications. Volume 181, No. 2, 317-324. (2010).

[16] M. F. Mahmood \& S. B. Qadri. "Modeling propagation of chirped solitons in an elliptically low birefringent single-mode optical fiber". Journal of Nonlinear Optical Physics and Materials. Volume 8, Number 4, 469-475. (1999). 
[17] Y. Pandir, Y. Gurefe, U. Kadak, E. Misirli. "Classification of exact solutions for some nonlinear partial differential equations with generalized evolution". Abstract and Applied Analysis. Volume 2012 (2012). Article ID 478531, 16 pages.

[18] M. Wadati, H. Segur \& M. J. Ablowitz. "A new Hamiltonian amplitude equation governing modulated wave instabilities". Journal of the Physical Society of Japan. Volume 61, Number 4, 1187-1193. (1992).

[19] W. P. Zhong. "Soliton propagation near zero-dispersion wavelength in birefringence fiber". Communications in Theoretical Physics. Volume 33, Number 1, 157-160. (2000).

[20] Q. Zhou, M. Mirzazadeh, M. Ekici \& A. Sonomezoglu. "Analytical study of solitons in non-Kerr nonlinear negative-indexed materials". To appear in Nonlinear Dynamics. DOI: 10.1007/s11071-016-2911-1 